Copyright (C) 2013 IEEE. Personal use of this material is permitted. Permission from IEEE must be obtained for all other uses, in any current or future media, including reprinting/republishing this material for advertising or promotional purposes, creating new collective works, for resale or redistribution to servers or lists, or reuse of any copyrighted component of this work in other works. 


\title{
Precoding Design for MIMO Relay Multicasting
}

\author{
Muhammad R. A. Khandaker, Student Member, IEEE, and Yue Rong, Senior Member, IEEE
}

\begin{abstract}
In this paper, we consider a two-hop multicasting multiple-input multiple-output (MIMO) relay system where one transmitter multicasts common message to multiple receivers with the aid of a relay node, and all nodes are equipped with multiple antennas. Joint transmit and relay precoding design problems are investigated for multicasting multiple data streams based on two design criteria. In the first scheme, we aim at minimizing the maximal mean-squared error (MSE) of the signal waveform estimation among all receivers subjecting to power constraints at the transmitter and the relay node. This problem is highly nonconvex with matrix variables and the exactly optimal solution is very hard to obtain. We develop an iterative algorithm to jointly optimize the transmitter, relay, and receiver matrices through solving convex subproblems. By exploiting the optimal structure of the relay precoding matrix, we then propose a low complexity solution which decouples the optimization of the transmitter and relay matrices under the (moderately) high first-hop signal-to-noise ratio (SNR) assumption. In the second scheme, we propose a total transmission power minimization strategy subjecting to quality-of-service $(\mathrm{QoS})$ constraints. By using the optimal structure of the relay precoding matrix and the (moderately) high first-hop SNR assumption, we show that this problem can be solved using the semidefinite programming (SDP) technique. Numerical simulations demonstrate the effectiveness of the proposed algorithms. Interestingly, we show that for the special case of single data stream multicasting, the relay precoding matrix optimization problem can be equivalently converted to the transmit beamforming problem for single-hop multicasting systems.
\end{abstract}

Index Terms-MIMO relay, multicasting, precoding.

\section{INTRODUCTION}

In many practical communication systems, one transmitter needs to send common message to a group of receivers simultaneously. These systems are referred to as multicast broadcasting or multicasting systems. The broadcasting nature of the wireless channel makes it naturally suitable for multicasting applications, since a single transmission may be simultaneously received by a number of users. Recently, wireless multicasting technology has triggered great interest among researchers across the world, due to the increasing demand for mobile applications such as streaming media, software updates, and location-based services involving group communications. The increasing popularity of live streaming, e.g. mobile TV or IPTV has also generated the need for efficient wireless solutions to support such applications and services. However, wireless channel is subject to signal fading. By exploiting the spatial diversity, multi-antenna techniques

Manuscript received November 16, 2012; revised March 18, 2013; accepted May 4, 2013. The associate editor coordinating the review of this paper and approving it for publication was Prof. Dusit Niyato.

M. R. A. Khandaker and Y. Rong are with the Department of Electrical and Computer Engineering, Curtin University, Bentley, WA 6102, Australia (e-mail: m.khandaker@postgrad.curtin.edu.au; y.rong@curtin.edu.au).

This work was supported under the Australian Research Council's Discovery Projects funding scheme (project numbers DP110100736, DP110102076). can be applied to combat channel fading [1]. Next generation wireless standards such as WiMAX 802.16m and 3GPP LTE-Advanced have already included technologies which enable better multicasting solutions based on multi-antenna and beamforming techniques [2].

The problem of designing optimal beamforming vectors for multicasting is hard in general, mainly due to its nonconvex nature. The authors in [3] have designed transmit beamformers for physical layer multicasting using rank relaxations. The information theoretic capacity of the multi-antenna multicasting channel is studied in [4] with a particular focus on the scaling of the capacity and achievable rates as the number of antennas and/or users approaches infinity. The asymptotic capacity limits of multi-antenna physical layer multicasting has been studied in [5] based on antenna subset selection. The effect of channel spatial correlation on the multicasting capacity has been investigated in [6]. The authors in [7] investigated transmit precoding design for multi-antenna multicasting systems where the channel state information (CSI) is obtained via limited feedback. The authors in [8] considered transmit covariance design for a secrecy rate maximization problem under a multicasting scenario, where a multi-antenna transmitter delivers a common confidential message to multiple single-antenna receivers in the presence of multiple multiantenna eavesdroppers.

The works in [3]-[8] solved the max-min signal-to-noise ratio (SNR)/rate beamforming problems with the aid of semidefinite relaxation (SDR) and rank-one approximation (which is suboptimal, in general). In [9], a stochastic beamforming strategy is proposed for multi-antenna physical layer multicasting where the randomization is guided by SDR, but without the need of rank-one approximation. The authors in [9] adopted an achievable rate perspective, and showed that the gaps between the rates of the proposed stochastic beamformers and the optimal multicasting capacity are no greater than 0.8314 $\mathrm{bps} / \mathrm{Hz}$. The fundamental limit of the max-min beamforming is that as the number of users grows to infinity, the achievable rate decreases to zero [4]. To cure this problem, a joint beamforming and admission control problem has been considered in [10] and [11], where a subset of users is selected so that certain quality-of-service (QoS) requirements can be satisfied. An iterative transmit beamforming strategy has been proposed in [11] for multiple cochannel multicasting groups to minimize the total power transmitted by the antenna array subjecting to signal-to-interference-plus-noise ratio (SINR) constraints at the receivers. It has been shown that the problem can be approximated by a second-order cone programming (SOCP) problem which does not require rank relaxations.

While the works in [3]-[11] investigated multicasting systems with single-antenna receivers, recently multi-antenna receivers have been considered for multicasting systems [12]- 
[14]. In particular, coordinated beamforming techniques have been investigated in [12] to facilitate physical layer multicasting with multi-antenna receivers, and a generalized form of block diagonalization has been proposed to make orthogonal transmissions to distinct multicasting antenna groups. In [13], non-iterative nearly optimal transmit beamformers are designed for wireless link layer multicasting with real-valued channels, and for complex-valued channels an upper-bound on the multicasting rate is derived. The scaling of the achievable rate for increasing number of users is investigated in [14] for multiple-input multiple-output (MIMO) multicasting where the transmission is coded at the application layer over a number of channel realizations.

The above works [2]-[14] considered single-hop multicasting systems. However, in the case of long transmitterreceiver distance, relay node(s) is necessary to efficiently combat the pathloss of wireless channel. The concept of multiuser peer-to-peer relay network has been generalized to that of a multi-group multicasting relay network in [15] and a distributed beamforming algorithm is proposed to minimize the total relay power where each node is equipped with a single antenna. In [16], the authors investigated multicast scheduling with multiple sessions and multiple channels where the base station may multicast data in two sessions using MIMO simultaneously through the same channel leading to a higher multicasting rate than single-session transmissions, and the users are allowed to cooperatively help each other on orthogonal channels. The authors in [17] studied the lowerbound for the outage probability of cooperative multi-antenna multicasting schemes based on the amplify-and-forward (AF) strategy where the users are equipped with a single antenna.

In this paper, we consider a two-hop multicasting MIMO relay system where one transmitter multicasts common message to multiple receivers with the aid of a relay node. The transmitter, relay node, and receivers are all equipped with multiple antennas. To the best of our knowledge, such two-hop multicasting MIMO relay system has not been investigated in existing works. For the sake of the implementation simplicity, we choose the AF relaying strategy. We consider the joint transmit and relay precoding design problem based on two criteria. In the first scheme, we aim at minimizing the maximal mean-squared error (MSE) of the signal waveform estimation among all receivers subjecting to power constraints at the transmitter and the relay node. This problem is highly nonconvex with matrix variables and the exactly optimal solution is very hard to obtain. We develop an iterative algorithm to jointly optimize the transmitter, relay, and receiver matrices through solving convex subproblems. By exploiting the optimal structure of the relay precoding matrix, we then propose a low complexity solution which decouples the optimization of the transmitter and relay matrices under the (moderately) high first-hop SNR assumption. In order to facilitate lowpower transmissions, in the second scheme, we propose a total transmission power minimization strategy subjecting to QoS constraints in terms of the MSE of the signal waveform estimation at each receiver. By using the optimal structure of the relay precoding matrix and the (moderately) high firsthop SNR assumption, we show that this problem can be solved using the semidefinite programming (SDP) technique. Numerical simulations demonstrate the effectiveness of the proposed algorithms. Note that both algorithms support multicasting multiple data streams in contrast to the existing single data stream multicasting schemes [2]-[16]. Interestingly, we show that for the special case of single data stream multicasting, the relay precoding matrix optimization problem can be equivalently converted to the transmit beamforming problem for single-hop multicasting systems. In this paper, for notational convenience, we consider a narrow-band singlecarrier system. However, our results can be straightforwardly generalized to each subcarrier of a broadband multi-carrier multicasting MIMO relay system.

The rest of this paper is organized as follows. In Section II, the system model of a two-hop multicasting MIMO relay network is introduced. The joint transmit and relay precoding matrices design algorithms are developed in Section III for the general case of multiple data streams multicasting. In Section IV, we study the joint transmit and relay beamforming for single data stream multicasting through two-hop MIMO relay systems. Section V shows the simulation results which justify the significance of the proposed algorithms under various scenarios. Conclusions are drawn in Section VI.

\section{SYSTEM MODEL}

We consider a two-hop MIMO multicasting system with $L$ receivers as illustrated in Fig. 1. The transmitter and the relay node are equipped with $N_{\mathrm{s}}$ and $N_{\mathrm{r}}$ antennas, respectively. For the sake of notational simplicity, we assume that each receiver has $N_{\mathrm{d}}$ antennas. The algorithms developed in this paper can be straightforwardly extended to multicasting systems where receivers have different number of antennas. The transmitter multicasts its information-carrying symbols to all receivers with the aid of a relay node. The direct links between the transmitter and the receivers are not considered since we assume that these direct links undergo much larger path attenuations compared with the links via the relay node.

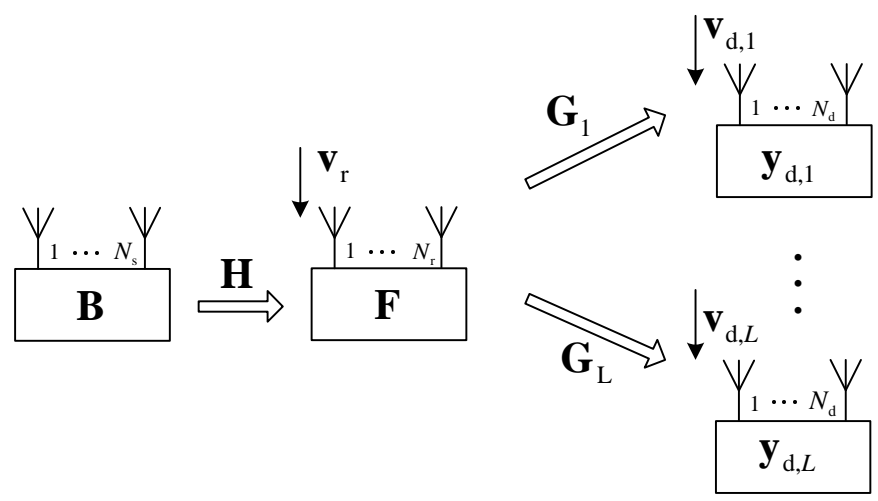

Fig. 1. Block diagram of a two-hop multicasting MIMO relay system.

We assume that the relay node works in half-duplex mode. Thus the communication between the transmitter and receivers is accomplished in two time slots. In the first time slot, the transmitter linearly precodes an $N_{\mathrm{b}} \times 1\left(N_{\mathrm{b}} \leq\right.$ $\left.\min \left(N_{\mathrm{s}}, N_{\mathrm{r}}, N_{\mathrm{d}}\right)\right)$ modulated signal vector $\mathbf{s}$ (common message to all receivers) by an $N_{\mathrm{s}} \times N_{\mathrm{b}}$ precoding matrix $\mathrm{B}$ 
and transmits the precoded vector $\mathbf{x}=\mathbf{B s}$ to the relay node. We assume that $\mathrm{E}\left[\mathbf{s s}^{H}\right]=\mathbf{I}_{N_{\mathrm{b}}}$, where $\mathrm{E}[\cdot]$ denotes statistical expectation, $(\cdot)^{H}$ stands for the matrix Hermitian transpose, and $\mathbf{I}_{n}$ is an $n \times n$ identity matrix. The received signal vector at the relay node is given by

$$
\mathbf{y}_{\mathrm{r}}=\mathbf{H B s}+\mathbf{v}_{\mathrm{r}}
$$

where $\mathbf{H}$ is the $N_{\mathrm{r}} \times N_{\mathrm{s}}$ MIMO channel matrix between the transmitter and the relay node, $\mathbf{y}_{\mathrm{r}}$ and $\mathbf{v}_{\mathrm{r}}$ are the $N_{\mathrm{r}} \times 1$ received signal and additive Gaussian noise vectors introduced at the relay node, respectively.

In the second time slot, the transmitter remains silent and the relay node multiplies (linearly precodes) the received signal vector $\mathbf{y}_{\mathrm{r}}$ by an $N_{\mathrm{r}} \times N_{\mathrm{r}}$ relay precoding matrix $\mathbf{F}$ and multicasts the precoded signal vector

$$
\mathbf{x}_{\mathrm{r}}=\mathbf{F} \mathbf{y}_{\mathrm{r}}
$$

to all $L$ receivers. From (1) and (2), the received signal vector at the $i$ th receiver can be written as

$$
\mathbf{y}_{\mathrm{d}, i}=\mathbf{G}_{i} \mathbf{F}\left(\mathbf{H B} \mathbf{s}+\mathbf{v}_{\mathbf{r}}\right)+\mathbf{v}_{\mathrm{d}, i} \triangleq \mathbf{A}_{i} \mathbf{s}+\mathbf{n}_{i}, i=1, \cdots, L
$$

where $\mathbf{G}_{i}$ is the $N_{\mathrm{d}} \times N_{\mathrm{r}}$ MIMO channel matrix between the relay node and the $i$ th receiver and $\mathbf{v}_{\mathrm{d}, i}$ is the additive Gaussian noise vector at the $i$ th receiver. Here $\mathbf{A}_{i} \triangleq \mathbf{G}_{i} \mathbf{F H B}$ is the equivalent MIMO channel between the transmitter and the $i$ th receiver, and $\mathbf{n}_{i} \triangleq \mathbf{G}_{i} \mathbf{F} \mathbf{v}_{\mathrm{r}}+\mathbf{v}_{\mathrm{d}, i}$ is the equivalent noise vector at the $i$ th receiver. All noises are independent and identically distributed (i.i.d.) complex circularly symmetric Gaussian noise with zero mean and unit variance.

We assume that all channels are quasi-static, i.e., the channel matrices $\mathbf{H}$ and $\mathbf{G}_{i}, i=1, \cdots, L$, are constant throughout a block of transmission. In practice, the CSI of $\mathbf{G}_{i}, i=1, \cdots, L$, can be obtained at the $i$ th receiver through standard training methods. The relay node can have the CSI of $\mathbf{H}$ through channel training, and obtain the CSI of $\mathbf{G}_{i}, i=1, \cdots, L$, by a feedback from the $i$ th receiver. The quasi-static channel model is valid in practice when the mobility among all communicating nodes is relatively slow. Therefore, we can obtain the necessary CSI with a reasonably high precision during the channel training period. The relay node calculates the optimal transmit matrix $\mathbf{B}$ and the relay matrix $\mathbf{F}$, and forwards $\mathbf{B}$ to the transmitter and forwards $\mathbf{B}$, $\mathbf{F}$, and $\mathbf{H}$ to all receivers ${ }^{1}$. Note that the transmitter does not need any channel knowledge and each receiver only needs the CSI of its own channel with the relay and that of the first-hop channel. This is a very important assumption for multicasting communication since in a multicasting scenario the receivers are distributed and cannot cooperate.

We aim at improving the system performance through optimizing the transmit and relay precoding matrices. Usually, the system performance is quantified by its QoS and the resources it consumes. The most commonly used QoS metrics

\footnotetext{
${ }^{1}$ This enables each receiver to calculate its receiver weight matrix. Alternatively, all receiver matrices can be calculated at the relay node, which then forwards the $i$ th receiver matrix to the $i$ th receiver. In this case, the receivers do not need to know $\mathbf{B}, \mathbf{F}$, and $\mathbf{H}$. However, the signalling overhead of the latter approach increases with the number of receivers, while the former approach does not.
}

include the MSE of the signal waveform estimation, bit-errorrate (BER), system capacity and the output SNR. Interestingly, the aforementioned QoS measures can be expressed in terms of MSE [18]. On the other hand, resources that a multicasting system consumes include the spectrum and transmission power. In the next section, we study two types of optimization problems for two-hop multicasting MIMO relay systems. The first problem deals with minimizing the MSE of the signal waveform estimation subjecting to transmission power constraints at the transmitter and the relay node, while the second problem investigates how to achieve given MSEs using a minimal possible total network transmission power.

\section{Proposed Transmitter and Relay Design ALGORITHMS}

Due to its simplicity, a linear receiver is used at each receiver to retrieve the transmitted signals. Denoting $\mathbf{W}_{i}$ as an $N_{\mathrm{d}} \times N_{\mathrm{b}}$ weight matrix at the $i$ th receiver, the estimated signal vector $\hat{\mathbf{s}}_{i}$ is given by

$$
\hat{\mathbf{s}}_{i}=\mathbf{W}_{i}^{H} \mathbf{y}_{\mathrm{d}, i}, \quad i=1, \cdots, L .
$$

From (4), the MSE of the signal waveform estimation at the $i$ th receiver is given by

$$
\begin{aligned}
E_{i}= & \operatorname{tr}\left(\mathrm{E}\left[\left(\hat{\mathbf{s}}_{i}-\mathbf{s}\right)\left(\hat{\mathbf{s}}_{i}-\mathbf{s}\right)^{H}\right]\right) \\
= & \operatorname{tr}\left(\left(\mathbf{W}_{i}^{H} \mathbf{A}_{i}-\mathbf{I}_{N_{\mathrm{b}}}\right)\left(\mathbf{W}_{i}^{H} \mathbf{A}_{i}-\mathbf{I}_{N_{\mathrm{b}}}\right)^{H}+\mathbf{W}_{i}^{H} \mathbf{C}_{i} \mathbf{W}_{i}\right) \\
& \quad i=1, \cdots, L
\end{aligned}
$$

where $\operatorname{tr}(\cdot)$ denotes matrix trace and $\mathbf{C}_{i} \triangleq \mathrm{E}\left[\mathbf{n}_{i} \mathbf{n}_{i}^{H}\right]=$ $\mathbf{G}_{i} \mathbf{F} \mathbf{F}^{H} \mathbf{G}_{i}^{H}+\mathbf{I}_{N_{\mathrm{d}}}$ is the covariance matrix of $\mathbf{n}_{i}$. Obviously, the power consumed by the transmitter is $\operatorname{tr}\left(\mathbf{B B}^{H}\right)$. And from (1) and (2), the transmission power consumed by the relay node is given by $\operatorname{tr}\left(\mathbf{F}\left(\mathbf{H B B}^{H} \mathbf{H}^{H}+\mathbf{I}_{N_{\mathrm{r}}}\right) \mathbf{F}^{H}\right)$. In the following, we consider two design strategies for optimizing the transmit and relay matrices. The first optimization strategy is to minimize the maximal MSE among all receivers subject to power constraints at the transmitter and the relay node. The second strategy minimizes the total network transmission power subject to QoS constraints.

\section{A. Min-Max MSE-Based Transmitter and Relay Design}

Given the power constraints at the transmitter and the relay node, we aim at minimizing the maximal MSE of the signal waveform estimations among all receivers. This problem formulation is important when the power consumption is a strict system constraint that cannot be relaxed. In this case, the transmitter, relay, and receiver matrices optimization problem can be formulated as

$$
\begin{aligned}
\min _{\mathbf{B}, \mathbf{F},\left\{\mathbf{W}_{i}\right\}} & \max _{i} E_{i} \\
\text { s.t. } & \operatorname{tr}\left(\mathbf{F}\left(\mathbf{H B B}^{H} \mathbf{H}^{H}+\mathbf{I}_{N_{\mathrm{r}}}\right) \mathbf{F}^{H}\right) \leq P_{\mathrm{r}} \\
& \operatorname{tr}\left(\mathbf{B B}^{H}\right) \leq P_{\mathbf{S}}
\end{aligned}
$$

where $\left\{\mathbf{W}_{i}\right\} \triangleq\left\{\mathbf{W}_{i}, i=1, \cdots, L\right\},(6 \mathrm{~b})$ and (6c) are the transmission power constraints at the relay node and the transmitter, respectively, and $P_{\mathrm{r}}>0, P_{\mathrm{s}}>0$ are the corresponding power budgets. In the following, we first develop an iterative algorithm to solve the problem (6) and then we propose a low complexity solution to this problem. 
1) Iterative Approach: This algorithm starts with random $\mathbf{F}$ and $\mathbf{B}$ satisfying (6b) and (6c). In each iteration, the transmitter, relay, and receiver matrices are updated alternatingly through solving convex subproblems. Firstly, with given $\mathbf{F}$ and $\mathbf{B}$, the optimal $\mathbf{W}_{i}, i=1, \cdots, L$, are obtained by solving the unconstrained convex problem of $\min _{\mathbf{W}_{i}} E_{i}$, since $E_{i}$ does not depend on $\mathbf{W}_{j}, j=1, \cdots, L, j \neq i$, and $\mathbf{W}_{i}$ does not appear in constraints (6b) and (6c). The solution is the wellknown linear minimal mean-squared error (MMSE) filter [19] and given by

$$
\mathbf{W}_{i}=\left(\mathbf{A}_{i} \mathbf{A}_{i}^{H}+\mathbf{C}_{i}\right)^{-1} \mathbf{A}_{i}, \quad i=1, \cdots, L
$$

where $(\cdot)^{-1}$ denotes matrix inversion.

Secondly, with given $\mathbf{W}_{i}, i=1, \cdots, L$, and $\mathbf{B}$, using the MSE expression in (5), the relay matrix optimization problem can be formulated as

$$
\begin{aligned}
\min _{\mathbf{F}} & \max _{i} \operatorname{tr}\left(\mathbf{W}_{i}^{H} \mathbf{G}_{i} \mathbf{F K F} \mathbf{F}^{H} \mathbf{G}_{i}^{H} \mathbf{W}_{i}-\mathbf{W}_{i}^{H} \mathbf{G}_{i} \mathbf{F H B}\right. \\
& \left.-\left(\mathbf{G}_{i} \mathbf{F H B}\right)^{H} \mathbf{W}_{i}\right)+\operatorname{tr}\left(\mathbf{W}_{i}^{H} \mathbf{W}_{i}+\mathbf{I}_{N_{\mathrm{r}}}\right) \\
\text { s.t. } & \operatorname{tr}\left(\mathbf{F} \mathbf{K} \mathbf{F}^{H}\right) \leq P_{\mathrm{r}}
\end{aligned}
$$

where $\mathbf{K} \triangleq \mathbf{H B B}^{H} \mathbf{H}^{H}+\mathbf{I}_{N_{\mathrm{r}}}$, and (8a) is obtained by substituting $\mathbf{A}_{i}$ into (5). Let us introduce

$$
\begin{aligned}
& \mathbf{F K F}^{H} \preccurlyeq \mathbf{\Phi} \\
& \mathbf{W}_{i}^{H} \mathbf{G}_{i} \mathbf{F} \mathbf{K F}^{H} \mathbf{G}_{i}^{H} \mathbf{W}_{i}-\mathbf{W}_{i}^{H} \mathbf{G}_{i} \mathbf{F H B} \\
& \quad-\left(\mathbf{G}_{i} \mathbf{F H B}\right)^{H} \mathbf{W}_{i} \preccurlyeq \mathbf{\Psi}_{i}, \quad i=1, \cdots, L
\end{aligned}
$$

and a real-valued slack variable $t_{\mathrm{r}}$, the problem (8) can be equivalently transformed to

$$
\begin{array}{ll}
\min _{t_{\mathrm{r}}, \mathbf{F},\left\{\mathbf{\Psi}_{i}\right\}, \boldsymbol{\Phi}} t_{\mathrm{r}} & \\
\text { s.t. } & \operatorname{tr}\left(\mathbf{\Psi}_{i}\right)+\operatorname{tr}\left(\mathbf{W}_{i}^{H} \mathbf{W}_{i}\right) \leq t_{\mathrm{r}}, \quad i=1, \cdots, L \\
& \left(\begin{array}{c}
\mathbf{\Psi}_{i}+\mathbf{W}_{i}^{H} \mathbf{G}_{i} \mathbf{F H B}+\left(\mathbf{G}_{i} \mathbf{F H B}\right)^{H} \mathbf{W}_{i} \mathbf{W}_{i}^{H} \mathbf{G}_{i} \mathbf{F} \\
\mathbf{F}^{H} \mathbf{G}_{i}^{H} \mathbf{W}_{i} \\
i=1, \cdots, L \\
\quad \operatorname{K}{ }^{-1}
\end{array}\right) \succcurlyeq 0 \\
& \operatorname{tr}(\mathbf{\Phi}) \leq P_{\mathrm{r}} \\
& \left(\begin{array}{cc}
\mathbf{\Phi} & \mathbf{F} \\
\mathbf{F}^{H} \mathbf{K}^{-1}
\end{array}\right) \succcurlyeq 0
\end{array}
$$

where $\left\{\boldsymbol{\Psi}_{i}\right\} \triangleq\left\{\boldsymbol{\Psi}_{i}, i=1, \cdots, L\right\}$ and we use the Schur complement to obtain (9c) and (9e). Here $\mathbf{A} \succcurlyeq 0$ indicates that matrix $\mathbf{A}$ is positive semidefinite (PSD), and $\mathbf{A} \preccurlyeq \mathbf{B}$ means $\mathbf{B}-\mathbf{A} \succcurlyeq 0$. The problem (9) is a convex SDP problem and can be efficiently solved by the disciplined convex programming toolbox CVX [23], where interior-point method-based solvers such as SeDuMi or SDPT3 are called internally.

Thirdly, to obtain the optimal $\mathbf{B}$ with given $\mathbf{F}$ and $\mathbf{W}_{i}, i=$ $1, \cdots, L$, we reformulate the problem (6) as

$$
\begin{aligned}
\min _{\mathbf{B}} & \max _{i} \operatorname{tr}\left(\mathbf{L}_{i} \mathbf{B B}^{H} \mathbf{L}_{i}^{H}-\mathbf{L}_{i} \mathbf{B}-\mathbf{B}^{H} \mathbf{L}_{i}^{H}\right) \\
& +\operatorname{tr}\left(\mathbf{W}_{i}^{H} \mathbf{G}_{i} \mathbf{F} \mathbf{F}^{H} \mathbf{G}_{i}^{H} \mathbf{W}_{i}+\mathbf{W}_{i}^{H} \mathbf{W}_{i}+\mathbf{I}_{N_{\mathrm{b}}}\right) \\
\text { s.t. } & \operatorname{tr}\left(\mathbf{F}\left(\mathbf{H B B}^{H} \mathbf{H}^{H}+\mathbf{I}_{N_{\mathrm{r}}}\right) \mathbf{F}^{H}\right) \leq P_{\mathrm{r}} \\
& \operatorname{tr}\left(\mathbf{B} \mathbf{B}^{H}\right) \leq P_{\mathrm{S}}
\end{aligned}
$$

where $\mathbf{L}_{i} \triangleq \mathbf{W}_{i}^{H} \mathbf{G}_{i} \mathbf{F H}, i=1, \cdots, L$. Let us introduce

$$
\begin{aligned}
& \mathbf{F H B B}{ }^{H} \mathbf{H}^{H} \mathbf{F}^{H} \preccurlyeq \mathbf{\Upsilon} \\
& \mathbf{B B}^{H} \preccurlyeq \boldsymbol{\Omega} \\
& \mathbf{L}_{i} \mathbf{B B}^{H} \mathbf{L}_{i}^{H}-\mathbf{L}_{i} \mathbf{B}-\mathbf{B}^{H} \mathbf{L}_{i}^{H} \preccurlyeq \boldsymbol{\Gamma}_{i}, \quad i=1, \cdots, L
\end{aligned}
$$

and a real-valued slack variable $t_{\mathrm{s}}$, the problem (10) can be equivalently transformed to

$$
\begin{aligned}
& \min _{t_{\mathrm{s}}, \mathbf{B},\left\{\boldsymbol{\Gamma}_{i}\right\}, \Upsilon, \boldsymbol{\Omega}} t_{\mathrm{S}} \\
& \text { s.t. } \operatorname{tr}\left(\boldsymbol{\Gamma}_{i}\right)+\operatorname{tr}\left(\mathbf{W}_{i}^{H} \mathbf{G}_{i} \mathbf{F} \mathbf{F}^{H} \mathbf{G}_{i}^{H} \mathbf{W}_{i}+\mathbf{W}_{i}^{H} \mathbf{W}_{i}\right) \leq t_{\mathrm{s}} \\
& i=1, \cdots, L \\
& \left(\begin{array}{cc}
\boldsymbol{\Gamma}_{i}+\mathbf{L}_{i} \mathbf{B}+\mathbf{B}^{H} \mathbf{L}_{i}^{H} & \mathbf{L}_{i} \mathbf{B} \\
\mathbf{B}^{H} \mathbf{L}_{i}^{H} & \mathbf{I}_{N_{\mathrm{b}}}
\end{array}\right) \succcurlyeq 0, \quad i=1, \cdots, L \\
& \operatorname{tr}(\mathbf{\Upsilon}) \leq P_{\mathrm{r}}-\operatorname{tr}\left(\mathbf{F} \mathbf{F}^{H}\right) \\
& \left(\begin{array}{cc}
\mathbf{\Upsilon} & \mathbf{F H B} \\
\mathbf{B}^{H} \mathbf{H}^{H} \mathbf{F}^{H} & \mathbf{I}_{N_{\mathrm{b}}}
\end{array}\right) \succcurlyeq 0 \\
& \operatorname{tr}(\boldsymbol{\Omega}) \leq P_{\mathrm{s}} \\
& \left(\begin{array}{cc}
\boldsymbol{\Omega} & \mathbf{B} \\
\mathbf{B}^{H} & \mathbf{I}_{N_{\mathrm{b}}}
\end{array}\right) \succcurlyeq 0
\end{aligned}
$$

where $\left\{\boldsymbol{\Gamma}_{i}\right\} \triangleq\left\{\boldsymbol{\Gamma}_{i}, i=1, \cdots, L\right\}$ and we use the Schur complement to obtain (11c), (11e), and (11g). The problem (11) is a convex SDP problem and we use the CVX software package [23] to solve the problem. Now the original transmitter, relay, and receiver matrices optimization problem (6) can be solved by an iterative technique as shown in Table I, where $\|\cdot\|_{1}$ denotes the matrix maximum absolute column sum norm, $\varepsilon$ is a small positive number close to zero and the superscript $(n)$ denotes the number of iterations.

TABLE I

PROCEDURE OF SOLVING THE PROBLEM (6) B Y THE ITERATIVE ALGORITHM

1) Initialize the algorithm with random $\mathbf{F}$ and $\mathbf{B}$ satisfying (6b) and (6c), for example, $\mathbf{B}^{(0)}=\sqrt{P_{\mathrm{s}} / N_{\mathrm{s}}} \mathbf{I}_{N_{\mathrm{s}}}$ and $\mathbf{F}^{(0)}=\sqrt{P_{\mathrm{r}} / \operatorname{tr}\left(\mathbf{K}^{(0)}\right)} \mathbf{I}_{N_{\mathrm{r}}}$; Set $n=0$

2) Update $\left\{\mathbf{W}_{i}^{(n)}\right\}$ using $\mathbf{B}^{(n)}$ and $\mathbf{F}^{(n)}$ as in (7).

3) Update $\mathbf{F}^{(n+1)}$ by solving the problem (9) using given $\left\{\mathbf{W}_{i}^{(n)}\right\}$ and $\mathbf{B}^{(n)}$.

4) Solve the problem (11) using known $\mathbf{F}^{(n+1)}$ and $\left\{\mathbf{W}_{i}^{(n)}\right\}$ to obtain $\mathbf{B}^{(n+1)}$.

5) If $\max \left\|\mathbf{B}^{(n+1)}-\mathbf{B}^{(n)}\right\|_{1} \leq \varepsilon$, then end. Otherwise, let $n:=n+1$ and go to step 2 .

In each iteration of the algorithm, the transmitter, relay, and receiver matrices are updated alternatingly through solving the corresponding subproblems. Since all subproblems are convex optimization problems, the conditional update of each matrix can not increase the objective function of each subproblem, and hence the objective function (6a). Therefore, each conditional update of $\mathbf{W}_{i}^{(n)}, i=1, \cdots, L, \mathbf{F}^{(n)}$, and $\mathbf{B}^{(n)}$ may either decrease or maintain but cannot increase the objective function (6a). Note that the constraints in the problem (6) are always satisfied with every conditional update. Thus a monotonic convergence of $\mathbf{W}_{i}^{(n)}, i=1, \cdots, L, \mathbf{F}^{(n)}$, and $\mathbf{B}^{(n)}$ towards (at least) a locally optimal solution follows directly from this observation. 
The major computational complexity of the iterative approach involves solving the SDP problems (9) and (11) in each iteration. Thus the overall computational complexity of the iterative algorithm is very high. In the following, we develop a simplified algorithm for the min-max MSE problem such that the nearly optimal transmitter and relay matrices can be designed with a significantly reduced computational complexity.

2) Simplified Approach: For any given $\mathbf{B}$ and F, by substituting (7) back into (5), we have

$$
\begin{aligned}
E_{i}= & \operatorname{tr}\left(\left[\mathbf{I}_{N_{\mathrm{b}}}+\mathbf{A}_{i}^{H} \mathbf{C}_{i}^{-1} \mathbf{A}_{i}\right]^{-1}\right) \\
= & \operatorname{tr}\left(\left[\mathbf{I}_{N_{\mathrm{b}}}+\mathbf{B}^{H} \mathbf{H}^{H} \mathbf{F}^{H} \mathbf{G}_{i}^{H}\left(\mathbf{G}_{i} \mathbf{F} \mathbf{F}^{H} \mathbf{G}_{i}^{H}+\mathbf{I}_{N_{\mathrm{d}}}\right)^{-1}\right.\right. \\
& \left.\left.\times \mathbf{G}_{i} \mathbf{F H B}\right]^{-1}\right), \quad i=1, \cdots, L
\end{aligned}
$$

Therefore, we can equivalently rewrite the problem (6) as

$$
\begin{array}{ll}
\min _{\mathbf{B}, \mathbf{F}} & \max _{i} \operatorname{tr}\left(\left[\mathbf{I}_{N_{\mathrm{b}}}+\mathbf{A}_{i}^{H} \mathbf{C}_{i}^{-1} \mathbf{A}_{i}\right]^{-1}\right) \\
\text { s.t. } & \operatorname{tr}\left(\mathbf{F}\left(\mathbf{H B B} \mathbf{B}^{H} \mathbf{H}^{H}+\mathbf{I}_{N_{\mathrm{r}}}\right) \mathbf{F}^{H}\right) \leq P_{\mathrm{r}} \\
& \operatorname{tr}\left(\mathbf{B B}^{H}\right) \leq P_{\mathrm{s}} .
\end{array}
$$

The min-max problem (13) is highly nonconvex with matrix variables, and an exactly optimal solution is very hard to obtain with a reasonable computational complexity (non-exhaustive searching). In the following, we propose a low complexity solution to the problem (13).

It can be shown similar to [20] that for any $\mathbf{B}$, the optimal $\mathbf{F}$ for the transmitter-relay- $i$ th receiver channel has a structure of $\mathbf{F}=\mathbf{T}_{i} \mathbf{D}^{H}$, where $\mathbf{D}=\left(\mathbf{H B B}{ }^{H} \mathbf{H}^{H}+\mathbf{I}_{N_{\mathbf{r}}}\right)^{-1} \mathbf{H B}$. Therefore, decomposition of $\mathbf{F}$ into

$$
\mathbf{F}=\mathbf{T D}^{H}
$$

is optimal for all receivers, which is also optimal with respect to the min-max problem (13). Interestingly, $\mathbf{D}$ can be viewed as the weight matrix of the linear MMSE receiver for the firsthop MIMO channel at the relay node given by (1), and $\mathbf{T}$ can be treated as the transmit precoding matrix for the effective second-hop MIMO multicasting system $\mathbf{y}_{i}=\mathbf{G}_{i} \mathbf{T x}+\mathbf{v}_{i}$, $i=1, \cdots, L$, where $\mathbf{x}$ is the transmitted signal vector and $\mathbf{v}_{i}$ is the noise vector.

Using the optimal $\mathbf{F}$ in (14), the MSE of the signal waveform estimation at the $i$ th receiver in (12) can be equivalently rewritten as the sum of two individual MSEs

$$
\begin{aligned}
E_{i}= & \operatorname{tr}\left(\left[\mathbf{I}_{N_{\mathrm{b}}}+\mathbf{B}^{H} \mathbf{H}^{H} \mathbf{H B}\right]^{-1}\right) \\
& +\operatorname{tr}\left(\left[\mathbf{R}^{-1}+\mathbf{T}^{H} \mathbf{G}_{i}^{H} \mathbf{G}_{i} \mathbf{T}\right]^{-1}\right), \quad i=1, \cdots, L
\end{aligned}
$$

where

$$
\mathbf{R}=\mathbf{B}^{H} \mathbf{H}^{H}\left(\mathbf{H B B}^{H} \mathbf{H}^{H}+\mathbf{I}_{N_{\mathrm{r}}}\right)^{-1} \mathbf{H B} .
$$

Note that the first term in (15) $\operatorname{tr}\left(\left[\mathbf{I}_{N_{\mathrm{b}}}+\mathbf{B}^{H} \mathbf{H}^{H} \mathbf{H B}\right]^{-1}\right)$ is actually the MSE of estimating the signal vector $\mathbf{s}$ from the received signal vector (1) at the relay node using the linear MMSE receiver $\mathbf{D}$, while the second term in (15) $\operatorname{tr}\left(\left[\mathbf{R}^{-1}+\mathbf{T}^{H} \mathbf{G}_{i}^{H} \mathbf{G}_{i} \mathbf{T}\right]^{-1}\right)$ can be viewed as the increment of the MSE introduced by the second-hop. Interestingly, matrix
$\mathbf{R}$ in (16) is in fact the covariance matrix of $\mathbf{z} \triangleq \mathbf{D}^{H} \mathbf{y}_{\mathbf{r}}$ as $\mathbf{R}=\mathrm{E}\left[\mathbf{z z}^{H}\right]=\mathbf{D}^{H} \mathrm{E}\left[\mathbf{y}_{\mathrm{r}} \mathbf{y}_{\mathrm{r}}^{H}\right] \mathbf{D}$. It can be seen from (15) that the effect of noise in the first-hop is reflected by $\mathbf{I}_{N_{\mathrm{b}}}$ in the first term and that of the second-hop is reflected by $\mathbf{R}^{-1}$ in the second term. Using the optimal structure of $\mathbf{F}$ in (14), the relay power consumption $\operatorname{tr}\left(\mathbf{F}\left(\mathbf{H B B}{ }^{H} \mathbf{H}^{H}+\mathbf{I}_{N_{\mathrm{r}}}\right) \mathbf{F}^{H}\right)$ is equivalent to $\operatorname{tr}\left(\mathbf{T R T}^{H}\right)$. Therefore, the problem (13) can be equivalently rewritten as

$$
\begin{gathered}
\min _{\mathbf{B}, \mathbf{T}} \max _{i} \operatorname{tr}\left(\left[\mathbf{I}_{N_{\mathrm{b}}}+\mathbf{B}^{H} \mathbf{H}^{H} \mathbf{H B}\right]^{-1}\right) \\
+\operatorname{tr}\left(\left[\mathbf{R}^{-1}+\mathbf{T}^{H} \mathbf{G}_{i}^{H} \mathbf{G}_{i} \mathbf{T}\right]^{-1}\right) \\
\text { s.t. } \operatorname{tr}\left(\mathbf{T} \mathbf{R} \mathbf{T}^{H}\right) \leq P_{\mathrm{r}} \\
\operatorname{tr}\left(\mathbf{B B}{ }^{H}\right) \leq P_{\mathrm{s}} .
\end{gathered}
$$

By applying the matrix inversion lemma $(\mathbf{A}+\mathbf{B C D})^{-1}=$ $\mathbf{A}^{-1}-\mathbf{A}^{-1} \mathbf{B}\left(\mathbf{D A}{ }^{-1} \mathbf{B}+\mathbf{C}^{-1}\right)^{-1} \mathbf{D A}^{-1}$, matrix $\mathbf{R}$ can be rewritten as

$$
\begin{aligned}
\mathbf{R} & =\mathbf{B}^{H} \mathbf{H}^{H}\left(\mathbf{I}_{N_{\mathrm{r}}}-\mathbf{H B}\left(\mathbf{B}^{H} \mathbf{H}^{H} \mathbf{H B}+\mathbf{I}_{N_{\mathrm{b}}}\right)^{-1} \mathbf{B}^{H} \mathbf{H}^{H}\right) \mathbf{H B} \\
& =\mathbf{B}^{H} \mathbf{H}^{H} \mathbf{H B}\left(\mathbf{B}^{H} \mathbf{H}^{H} \mathbf{H B}+\mathbf{I}_{N_{\mathrm{b}}}\right)^{-1} .
\end{aligned}
$$

An interesting observation from (18) is that with increasing first-hop SNR, the term $\mathbf{B}^{H} \mathbf{H}^{H} \mathbf{H B}$ approaches to infinity. And at a (moderately) high SNR level, there is $\mathbf{B}^{H} \mathbf{H}^{H} \mathbf{H B} \gg$ $\mathbf{I}_{N_{\mathrm{b}}}$. Here for matrices $\mathbf{X}$ and $\mathbf{Y}, \mathbf{X} \gg \mathbf{Y}$ indicates that the eigenvalues of $(\mathbf{X}-\mathbf{Y})$ are much greater than zero. Thus we can strictly upper-bound $\mathbf{R}$ as $\mathbf{I}_{N_{\mathrm{b}}}$ for the high SNR case [21]. As a consequence, $\operatorname{tr}\left(\left[\mathbf{R}^{-1}+\mathbf{T}^{H} \mathbf{G}_{i}^{H} \mathbf{G}_{i} \mathbf{T}\right]^{-1}\right)$ in (17a) is upper-bounded by $\operatorname{tr}\left(\left[\mathbf{I}_{N_{\mathrm{b}}}+\mathbf{T}^{H} \mathbf{G}_{i}^{H} \mathbf{G}_{i} \mathbf{T}\right]^{-1}\right)$, $i=1, \cdots, L$, as shown below: $\mathbf{R} \prec \mathbf{I}_{N_{\mathrm{b}}} \longrightarrow \mathbf{R}^{-1} \succ$ $\mathbf{I}_{N_{\mathrm{b}}} \longrightarrow \mathbf{R}^{-1}+\mathbf{T}^{H} \mathbf{G}_{i}^{H} \mathbf{G}_{i} \mathbf{T} \succ \mathbf{I}_{N_{\mathrm{b}}}+\mathbf{T}^{H} \mathbf{G}_{i}^{H} \mathbf{G}_{i} \mathbf{T} \longrightarrow$ $\left[\mathbf{R}^{-1}+\mathbf{T}^{H} \mathbf{G}_{i}^{H} \mathbf{G}_{i} \mathbf{T}\right]^{-1} \prec\left[\mathbf{I}_{N_{\mathrm{b}}}+\mathbf{T}^{H} \mathbf{G}_{i}^{H} \mathbf{G}_{i} \mathbf{T}\right]^{-1} \longrightarrow$ $\operatorname{tr}\left(\left[\mathbf{R}^{-1}+\mathbf{T}^{H} \mathbf{G}_{i}^{H} \mathbf{G}_{i} \mathbf{T}\right]^{-1}\right)<\operatorname{tr}\left(\left[\mathbf{I}_{N_{\mathrm{b}}}+\mathbf{T}^{H} \mathbf{G}_{i}^{H} \mathbf{G}_{i} \mathbf{T}\right]^{-1}\right)$. Here for matrices $\mathbf{A}$ and $\mathbf{B}, \mathbf{A} \prec \mathbf{B}$ means $\mathbf{B}-\mathbf{A}$ is a positive definite matrix, and $\mathbf{A} \succ \mathbf{B}$ means $\mathbf{A}-\mathbf{B}$ is a positive definite matrix. The tightness of this bound increases with the first-hop SNR. Therefore, the problem (17) can be approximated as

$$
\begin{aligned}
\min _{\mathbf{B}, \mathbf{T}} \max _{i} \operatorname{tr}\left(\left[\mathbf{I}_{N_{\mathrm{b}}}+\mathbf{B}^{H} \mathbf{H}^{H} \mathbf{H B}\right]^{-1}\right) \\
+\operatorname{tr}\left(\left[\mathbf{I}_{N_{\mathrm{b}}}+\mathbf{T}^{H} \mathbf{G}_{i}^{H} \mathbf{G}_{i} \mathbf{T}\right]^{-1}\right) \\
\text { s.t. } \operatorname{tr}\left(\mathbf{T} \mathbf{T}^{H}\right) \leq P_{\mathrm{r}} \\
\operatorname{tr}\left(\mathbf{B} \mathbf{B}^{H}\right) \leq P_{\mathbf{s}} .
\end{aligned}
$$

We would like to mention that since $\operatorname{tr}\left(\mathbf{T T}^{H}\right)>\operatorname{tr}\left(\mathbf{T R T}^{H}\right)$, if $\operatorname{tr}\left(\mathbf{T} \mathbf{T}^{H}\right)=p$, then $\operatorname{tr}\left(\mathbf{T R} \mathbf{T}^{H}\right)<p$. This indicates that due to the approximation in (19b), the transmission power available at the relay node is not fully utilized in the case of the low first-hop SNR. We can simply scale the relay matrix obtained from solving the problem (19) to compensate such slight loss and make the best use of the available power budget at the relay node. We can justify the need for the scaling operation as follows. Assuming that $\mathbf{T}_{1}=c \mathbf{T}_{2}$ with $c>1$, it can be seen from (19a) that $\operatorname{tr}\left(\left[\mathbf{I}_{N_{\mathrm{b}}}+\mathbf{T}_{1}^{H} \mathbf{G}_{i}^{H} \mathbf{G}_{i} \mathbf{T}_{1}\right]^{-1}\right)=$ $\operatorname{tr}\left(\left[\mathbf{I}_{N_{\mathrm{b}}}+c^{2} \mathbf{T}_{2}^{H} \mathbf{G}_{i}^{H} \mathbf{G}_{i} \mathbf{T}_{2}\right]^{-1}\right)<\operatorname{tr}\left(\left[\mathbf{I}_{N_{\mathrm{b}}}+\mathbf{T}_{2}^{H} \mathbf{G}_{i}^{H} \mathbf{G}_{i} \mathbf{T}_{2}\right]^{-1}\right)$. 
Therefore, the entire power should be utilized to minimize (19a).

Interestingly, it can be seen from the problem (19) that $\mathbf{T}$ does not affect the first term of the objective function (19a) and $\mathbf{B}$ is irrelevant to the second term of (19a). This fact implies that the objective function (19a) and the constraints (19b) and (19c) are decoupled with respect to the optimization variables $\mathbf{B}$ and $\mathbf{T}$. In this case, matrix $\mathbf{B}$ can be determined independent of $\mathbf{T}$, and vice-versa, which greatly simplifies the design of the transmit and relay matrices. Therefore, with the (relatively) high first-hop SNR assumption, the problem (19) can be decomposed into the following transmit precoding matrix optimization problem

$$
\begin{aligned}
& \min _{\mathbf{B}} \operatorname{tr}\left(\left[\mathbf{I}_{N_{\mathrm{b}}}+\mathbf{B}^{H} \mathbf{H}^{H} \mathbf{H B}\right]^{-1}\right) \\
& \text { s.t. } \operatorname{tr}\left(\mathbf{B B}^{H}\right) \leq P_{\mathbf{s}}
\end{aligned}
$$

and the relay precoding matrix optimization problem

$$
\begin{aligned}
& \min _{\mathbf{T}} \max _{i} \operatorname{tr}\left(\left[\mathbf{I}_{N_{\mathrm{b}}}+\mathbf{T}^{H} \mathbf{G}_{i}^{H} \mathbf{G}_{i} \mathbf{T}\right]^{-1}\right) \\
& \text { s.t. } \operatorname{tr}\left(\mathbf{T} \mathbf{T}^{H}\right) \leq P_{\mathrm{r}} .
\end{aligned}
$$

Let $\mathbf{H}=\mathbf{U}_{\mathrm{h}} \boldsymbol{\Lambda}_{\mathrm{h}} \mathbf{V}_{\mathrm{h}}^{H}$ denote the singular value decomposition (SVD) of $\mathbf{H}$, where the dimensions of $\mathbf{U}_{\mathrm{h}}, \boldsymbol{\Lambda}_{\mathrm{h}}$, and $\mathbf{V}_{\mathrm{h}}$ are $N_{\mathrm{r}} \times N_{\mathrm{r}}, N_{\mathrm{r}} \times N_{\mathrm{s}}$, and $N_{\mathrm{S}} \times N_{\mathrm{s}}$, respectively. We assume that the main diagonal elements of $\boldsymbol{\Lambda}_{\mathrm{h}}$ are arranged in decreasing order. According to Lemma 2 in [20], the transmitter optimization problem (20) has a closed form solution with the optimal structure of $\mathbf{B}$ given by

$$
\mathbf{B}=\mathbf{V}_{\mathrm{h}, 1} \boldsymbol{\Lambda}_{\mathrm{b}}
$$

where $\mathbf{V}_{\mathrm{h}, 1}$ contains the leftmost $N_{\mathrm{b}}$ columns of $\mathbf{V}_{\mathrm{h}}$ and $\boldsymbol{\Lambda}_{\mathrm{b}}$ is an $N_{\mathrm{b}} \times N_{\mathrm{b}}$ diagonal power loading matrix. Substituting the optimal $\mathbf{B}$ in (22) back into (20) and using the Lagrangian multiplier method [22], we find that the $i$ th diagonal element of $\boldsymbol{\Lambda}_{\mathrm{b}}$ is given by

$$
\lambda_{\mathrm{b}, i}=\left[\frac{1}{\lambda_{\mathrm{h}, i}}\left(\sqrt{\lambda_{\mathrm{h}, i} / \mu}-1\right)^{+}\right]^{\frac{1}{2}}, \quad i=1, \cdots, N_{\mathrm{b}}
$$

where $(x)^{+} \triangleq \max (x, 0), \lambda_{\mathrm{h}, i}$ is the $i$ th diagonal element of $\boldsymbol{\Lambda}_{\mathrm{h}}$, and $\mu>0$ is the Lagrangian multiplier and the solution to the nonlinear equation of $\sum_{i=1}^{N_{\mathrm{b}}} \frac{1}{\lambda_{\mathrm{h}, i}}\left(\sqrt{\lambda_{\mathrm{h}, i} / \mu}-1\right)^{+}=P_{\mathrm{s}}$.

By introducing $\mathbf{T} \mathbf{T}^{H} \triangleq \mathbf{Q}$, and using the matrix identity $\operatorname{tr}\left(\left[\mathbf{I}_{m}+\mathbf{A}_{m \times n} \mathbf{B}_{n \times m}\right]^{-1}\right)=\operatorname{tr}\left(\left[\mathbf{I}_{n}+\mathbf{B}_{n \times m} \mathbf{A}_{m \times n}\right]^{-1}\right)+$ $m-n$, the problem (21) can be equivalently rewritten as

$$
\begin{aligned}
& \min _{\mathbf{Q}} \max _{i} \operatorname{tr}\left(\left[\mathbf{I}_{N_{\mathrm{d}}}+\mathbf{G}_{i} \mathbf{Q G}_{i}^{H}\right]^{-1}\right)+N_{\mathrm{b}}-N_{\mathrm{d}} \\
& \text { s.t. } \operatorname{tr}(\mathbf{Q}) \leq P_{\mathrm{r}} \\
& \quad \mathbf{Q} \succcurlyeq 0
\end{aligned}
$$

By introducing $\left[\mathbf{I}_{N_{\mathrm{d}}}+\mathbf{G}_{i} \mathbf{Q G}_{i}^{H}\right]^{-1} \preccurlyeq \mathbf{Y}_{i}, i=1, \cdots, L$, and a real-valued slack variable $t$, the problem (23) can be equivalently transformed to

$$
\begin{aligned}
\min _{t, \mathbf{Q},\left\{\mathbf{Y}_{i}\right\}} & t \\
\text { s.t. } & \operatorname{tr}\left(\mathbf{Y}_{i}\right) \leq t, \quad i=1, \cdots, L \\
& \operatorname{tr}(\mathbf{Q}) \leq P_{\mathrm{r}} \\
& \left(\begin{array}{l}
\mathbf{Y}_{i} \quad \mathbf{I}_{N_{\mathrm{d}}} \\
\mathbf{I}_{N_{\mathrm{d}}} \mathbf{I}_{N_{\mathrm{d}}}+\mathbf{G}_{i} \mathbf{Q} \mathbf{G}_{i}^{H}
\end{array}\right) \succcurlyeq 0, \quad i=1, \cdots, L \\
& \mathbf{Q} \succcurlyeq 0
\end{aligned}
$$

where $\left\{\mathbf{Y}_{i}\right\} \triangleq\left\{\mathbf{Y}_{i}, i=1, \cdots, L\right\}$ and we use the Schur complement to obtain (24d). Note that in the above formulation, $t$ provides an MSE upper-bound for the relay-receiver channels. The problem (24) is an SDP problem which can be efficiently solved by the interior-point method at a maximal complexity order of $\mathcal{O}\left(\left(N_{\mathrm{r}}^{2}+L+1\right)^{3.5}\right)$ [24]. Since most of the computation task in solving the problem (20) involves performing SVD and calculating the power loading parameters, the computation overhead is negligible compared with that of solving the problem (24). Note that the problem (19) can also be directly formulated as an SDP problem which can be solved using interior point-based solvers at a complexity order that is at most $\mathcal{O}\left(\left(N_{\mathrm{s}}^{2}+N_{\mathrm{r}}^{2}+L+2\right)^{3.5}\right)$. Therefore, solving the decoupled transmit and relay precoding problems (20) and (21) has a much smaller computational complexity compared with directly solving the problem (19), especially in the case of large $N_{\mathrm{s}}$.

\section{B. Minimal Total Power-Based Transmitter and Relay Design}

In this scheme, we aim at minimizing the total transmitter and relay power consumption satisfying the target QoS requirements at all receivers. This criterion is important when certain QoS level must be maintained at each receiver. We choose the MSE of the signal waveform estimation at the receiver as the QoS metric. The multicasting MIMO relay system tries to satisfy the given required QoS (maximal allowable MSE) with the minimal total transmission power. Thus the optimization problem can be written as

$$
\begin{aligned}
& \min _{\mathbf{B}, \mathbf{F}} \operatorname{tr}\left(\mathbf{F}\left(\mathbf{H B B} \mathbf{B}^{H} \mathbf{H}^{H}+\mathbf{I}_{N_{\mathrm{r}}}\right) \mathbf{F}^{H}\right)+\operatorname{tr}\left(\mathbf{B B}^{H}\right) \\
& \text { s.t. } \operatorname{tr}\left(\left[\mathbf{I}_{N_{\mathrm{b}}}+\mathbf{A}_{i}^{H} \mathbf{C}_{i}^{-1} \mathbf{A}_{i}\right]^{-1}\right) \leq \varepsilon_{i}, \quad i=1, \cdots, L
\end{aligned}
$$

where $\varepsilon_{i}$ is the maximal allowable MSE at the $i$ th receiver. In almost all situations, the problem (25) is feasible for $\varepsilon_{i} \geq 0, i=1, \cdots, L$. The only exception is when the channel matrices are 'ill', for example $\mathbf{H}=0$, or $\mathbf{G}_{i}=0$. Obviously, since channel matrices are random, the probability of having ill channel matrices is zero in practice. Similar to the problem (13), the problem (25) is highly nonconvex with matrix variables.

It can be shown similar to [20] that the optimal structure of $\mathbf{F}$ for the problem (25) is given by (14). Then, the problem (25) can be equivalently converted to the following problem

$$
\begin{aligned}
& \min _{\mathbf{B}, \mathbf{T}} \operatorname{tr}\left(\mathbf{T R} \mathbf{T}^{H}\right)+\operatorname{tr}\left(\mathbf{B B} \mathbf{B}^{H}\right) \\
& \text { s.t. } \operatorname{tr}\left(\left[\mathbf{I}_{N_{\mathrm{b}}}+\mathbf{B}^{H} \mathbf{H}^{H} \mathbf{H B}\right]^{-1}\right) \\
& \quad+\operatorname{tr}\left(\left[\mathbf{R}^{-1}+\mathbf{T}^{H} \mathbf{G}_{i}^{H} \mathbf{G}_{i} \mathbf{T}\right]^{-1}\right) \leq \varepsilon_{i}, i=1, \cdots, L
\end{aligned}
$$


Similar to Section III-A, we have $\operatorname{tr}\left(\left[\mathbf{R}^{-1}+\mathbf{T}^{H} \mathbf{G}_{i}^{H} \mathbf{G}_{i} \mathbf{T}\right]^{-1}\right)$ $\leq \operatorname{tr}\left(\left[\mathbf{I}_{N_{\mathrm{b}}}+\mathbf{T}^{H} \mathbf{G}_{i}^{H} \mathbf{G}_{i} \mathbf{T}\right]^{-1}\right)$, and at a relatively high firsthop SNR, the problem (26) can be approximated (relaxed) to the problem of

$$
\begin{aligned}
& \min _{\mathbf{B}, \mathbf{T}} \operatorname{tr}\left(\mathbf{T} \mathbf{T}^{H}\right)+\operatorname{tr}\left(\mathbf{B} \mathbf{B}^{H}\right) \\
& \text { s.t. } \operatorname{tr}\left(\left[\mathbf{I}_{N_{\mathrm{b}}}+\mathbf{B}^{H} \mathbf{H}^{H} \mathbf{H B}\right]^{-1}\right) \\
& \quad+\operatorname{tr}\left(\left[\mathbf{I}_{N_{\mathrm{b}}}+\mathbf{T}^{H} \mathbf{G}_{i}^{H} \mathbf{G}_{i} \mathbf{T}\right]^{-1}\right) \leq \varepsilon_{i}, i=1, \cdots, L .
\end{aligned}
$$

By introducing $\mathbf{B B}^{H} \triangleq \mathbf{P}$ and $\left[\mathbf{I}_{N_{\mathrm{r}}}+\mathbf{H} \mathbf{P} \mathbf{H}^{H}\right]^{-1} \preccurlyeq \mathbf{X}$, the problem (27) can be transformed to the following SDP problem

$$
\begin{aligned}
& \min _{\mathbf{P}, \mathbf{Q}, \mathbf{X},\left\{\mathbf{Y}_{i}\right\}} \operatorname{tr}(\mathbf{P})+\operatorname{tr}(\mathbf{Q}) \\
& \text { s.t. } \operatorname{tr}(\mathbf{X})+\operatorname{tr}\left(\mathbf{Y}_{i}\right) \leq \varepsilon_{i}+N_{\mathrm{d}}+N_{\mathrm{r}}-2 N_{\mathrm{b}} \\
& i=1, \cdots, L \\
& \left(\begin{array}{cc}
\mathbf{X} & \mathbf{I}_{N_{\mathrm{r}}} \\
\mathbf{I}_{N_{\mathrm{r}}} \mathbf{I}_{N_{\mathrm{r}}}+\mathbf{H P H}
\end{array}\right) \succcurlyeq 0 \\
& \left(\begin{array}{cc}
\mathbf{Y}_{i} & \mathbf{I}_{N_{\mathrm{d}}} \\
\mathbf{I}_{N_{\mathrm{d}}} \mathbf{I}_{N_{\mathrm{d}}}+\mathbf{G}_{i} \mathbf{Q} \mathbf{G}_{i}^{H}
\end{array}\right) \succcurlyeq 0, i=1, \cdots, L \\
& \mathbf{P} \succcurlyeq 0, \quad \mathbf{Q} \succcurlyeq 0
\end{aligned}
$$

where the Schur complement is used to obtain (28c). Note that unlike the problem (19), the problem (28) is not decoupled due to the constraint (28b) which couples $\operatorname{tr}(\mathbf{X})$ and $\operatorname{tr}\left(\mathbf{Y}_{i}\right)$. However, we can use the CVX software package [23] to solve the SDP problem (28) at a complexity order that is at most $\mathcal{O}\left(\left(N_{\mathrm{s}}^{2}+N_{\mathrm{r}}^{2}+L\right)^{3.5}\right)$ and is usually much less [3].

We would like to mention that for a point-to-point twohop MIMO relay system, it has been shown in [21] through numerical examples that the high first-hop SNR approximation provides negligible performance loss in all SNR range in comparison to the optimal designs. For the multicasting MIMO relay system addressed in this paper, the exactly optimal solution for the transmit and relay precoding matrices are intractable. However, by using the high first-hop SNR approximation, the nearly optimal transmit and relay matrices can be designed with a significantly reduced computational complexity.

Although a two-hop multicasting MIMO relay system is considered in this paper, the simplified algorithm proposed in Section III.A.2 and the algorithm developed in Section III.B can be straightforwardly extended to the multi-hop scenario with multiple relays in series by using the high SNR upperbound technique, as in [20].

\section{TRAnSmitTER AND RELAy Optimization FOR Single Data Stream Multicasting}

The transmit and relay precoding matrices developed in the last section are applicable for the general case of multidatastream multicasting. In this section, we derive the solution for the min-max MSE and the total power minimization problems for the special case of single data stream multicasting in two-hop MIMO relay systems. In the case where the transmitter multicasts a single data stream $s$, i.e., $N_{\mathrm{b}}=1$, the received signal vector at the $i$ th receiver can be written as

$$
\mathbf{y}_{\mathrm{d}, i}=\mathbf{G}_{i} \mathbf{F}\left(\mathbf{H b} s+\mathbf{v}_{\mathrm{r}}\right)+\mathbf{v}_{\mathrm{d}, i}, \quad i=1, \cdots, L
$$

where $\mathbf{b}$ is the $N_{\mathrm{s}} \times 1$ transmit beamforming vector. In the following, we solve the min-max MSE and the total network transmission power minimization problems for the system in (29). In particular, we establish an interesting link between the relay precoding matrix in a two-hop MIMO multicasting system and the transmit beamformer in a singlehop multicasting system.

\section{A. Min-Max MSE-Based Transmitter and Relay Design}

Using a linear MMSE receiver $\mathbf{w}_{i}\left((7)\right.$ with $\left.N_{\mathrm{b}}=1\right)$ at the $i$ th receiver to estimate $s$ from (29), it can be shown similar to (12) that the MSE of the signal waveform estimation can be written as

$$
\begin{aligned}
e_{i}= & {\left[1+\left(\mathbf{G}_{i} \mathbf{F H b}\right)^{H}\left(\mathbf{G}_{i} \mathbf{F F} \mathbf{F}^{H} \mathbf{G}_{i}^{H}+\mathbf{I}_{N_{\mathrm{d}}}\right)^{-1} \mathbf{G}_{i} \mathbf{F H b}\right]^{-1} } \\
& i=1, \cdots, L .
\end{aligned}
$$

Similar to Section III-A, the worst-case MSE minimization problem for a single data stream multicasting MIMO relay system can be formulated as

$$
\begin{aligned}
& \left.\min _{\mathbf{b}, \mathbf{F}} \max _{i}\left[1+\left(\mathbf{G}_{i} \mathbf{F H} \mathbf{b}\right)^{H}\left(\mathbf{G}_{i} \mathbf{F} \mathbf{F}^{H} \mathbf{G}_{i}^{H}+\mathbf{I}_{N_{\mathrm{d}}}\right)^{-1} \mathbf{G}_{i} \mathbf{F H} \mathbf{b}\right] \overline{3} 1 \mathbf{a}\right) \\
& \text { s.t. } \operatorname{tr}\left(\mathbf{F}\left(\mathbf{H b b}^{H} \mathbf{H}^{H}+\mathbf{I}_{N_{\mathrm{r}}}\right) \mathbf{F}^{H}\right) \leq P_{\mathrm{r}} \\
& \quad \mathbf{b}^{H} \mathbf{b} \leq P_{\mathrm{s}} .
\end{aligned}
$$

We would like to mention that for the single data stream multicasting case, minimizing the MSE of the signal waveform estimation is equivalent to maximizing the receive SNR, and thus, the transmitter-receiver mutual information (MI).

Let $\mathbf{G}_{i}=\mathbf{U}_{i} \boldsymbol{\Lambda}_{i} \mathbf{V}_{i}^{H}$ denote the SVD of $\mathbf{G}_{i}, i=1, \cdots, L$, where the dimensions of $\mathbf{U}_{i}, \boldsymbol{\Lambda}_{i}$, and $\mathbf{V}_{i}$ are $N_{\mathrm{d}} \times N_{\mathrm{d}}$, $N_{\mathrm{d}} \times N_{\mathrm{r}}$, and $N_{\mathrm{r}} \times N_{\mathrm{r}}$, respectively. We assume that the main diagonal elements of $\boldsymbol{\Lambda}_{i}$ are arranged in decreasing order. According to the unified framework developed in [25], the optimal transmitter forms a "beam" along the direction of the strongest singular value of the transmitter-relay channel and hence, the transmit beamforming vector is given by

$$
\mathbf{b}=\mathbf{v}_{\mathrm{h}, 1} \sqrt{P_{\mathrm{s}}}
$$

where $\mathbf{v}_{\mathrm{h}, 1}$ is the leftmost column of $\mathbf{V}_{\mathrm{h}}$. Moreover, the optimal relay precoding matrix is given by $\mathbf{F}=\alpha_{i} \mathbf{v}_{i, 1} \mathbf{u}_{\mathrm{h}, 1}^{H}$ for the transmitter-relay-ith receiver channel [25], where $\alpha_{i}, i=$ $1, \cdots, L$, is the power loading factor at the relay node, $\mathbf{u}_{\mathrm{h}, 1}$ and $\mathbf{v}_{i, 1}$ are the leftmost columns of $\mathbf{U}_{\mathrm{h}}$ and $\mathbf{V}_{i}, i=1, \cdots, L$, respectively.

Although the optimal $\mathbf{F}$ for the $i$ th receiver depends on $\mathbf{G}_{i}$, it can be seen from the discussion above that $\mathbf{F}$ has the generic optimal structure of

$$
\mathbf{F}=\mathbf{f u}_{\mathrm{h}, 1}^{H}
$$

which is optimal for all receivers. Here vector $\mathbf{f}$ remains to be optimized. Using (32) and (33), the MSE in (30) can be 
rewritten as

$$
\begin{aligned}
e_{i}= & {\left[1+P_{\mathrm{s}} \lambda_{\mathrm{h}, 1}^{2} \mathbf{f}^{H} \mathbf{G}_{i}^{H}\left(\mathbf{G}_{i} \mathrm{ff}^{H} \mathbf{G}_{i}^{H}+\mathbf{I}_{N_{\mathrm{d}}}\right)^{-1} \mathbf{G}_{i} \mathbf{f}\right]^{-1} } \\
& i=1, \cdots, L
\end{aligned}
$$

where $\lambda_{\mathrm{h}, 1}$ is the largest singular value of $\mathbf{H}$. From (32) and (33), the relay transmit power (31b) becomes

$$
\operatorname{tr}\left(P_{\mathrm{s}} \lambda_{\mathrm{h}, 1}^{2} \mathrm{ff}^{H}+\mathrm{ff}^{H}\right)=\mathbf{f}^{H} \mathbf{f}\left(P_{\mathrm{s}} \lambda_{\mathrm{h}, 1}^{2}+1\right) .
$$

Applying the matrix inversion lemma, (34) can be equivalently rewritten as

$$
\begin{aligned}
e_{i} & =\left[1+\frac{P_{\mathrm{s}} \lambda_{\mathrm{h}, 1}^{2} \mathbf{f}^{H} \mathbf{G}_{i}^{H} \mathbf{G}_{i} \mathbf{f}}{\mathbf{f}^{H} \mathbf{G}_{i}^{H} \mathbf{G}_{i} \mathbf{f}+1}\right]^{-1} \\
& =\left[1+P_{\mathrm{s}} \lambda_{\mathrm{h}, 1}^{2}-\frac{P_{\mathrm{s}} \lambda_{\mathrm{h}, 1}^{2}}{\mathbf{f}^{H} \mathbf{G}_{i}^{H} \mathbf{G}_{i} \mathbf{f}+1}\right]^{-1}, i=1, \cdots, L
\end{aligned}
$$

Note that minimizing the MSE in (35) is equivalent to maximizing $\mathbf{f}^{H} \mathbf{G}_{i}^{H} \mathbf{G}_{i} \mathbf{f}$ which in fact determines the SNR at the $i$ th receiver in a single-hop (relay-receiver channel in this case) MIMO multicasting system. Therefore, the min-max MSE problem (31) is equivalent to the following max-min SNR optimization problem

$$
\begin{gathered}
\max _{\mathbf{f}} \min _{i} \mathbf{f}^{H} \mathbf{G}_{i}^{H} \mathbf{G}_{i} \mathbf{f} \\
\text { s.t. } \mathbf{f}^{H} \mathbf{f} \leq \frac{P_{\mathrm{r}}}{P_{\mathrm{s}} \lambda_{\mathrm{h}, 1}^{2}+1} .
\end{gathered}
$$

Interestingly, the problem (36) is equivalent to a max-min transmit beamforming problem for a single-hop multicasting system, which can be efficiently solved using standard techniques such as rank relaxations at a maximal complexity order of $\mathcal{O}\left(\left(N_{\mathrm{r}}^{2}+L+1\right)^{3.5}\right)$ [3].

\section{B. Minimal Total Power-Based Transmitter and Relay Design}

For single data stream multicasting, the design of the transmit beamformer and the relay precoding matrix that minimize the total network transmission power, subjecting to constraints on the MSE of the signal waveform estimation at each user (30), can be written as the following problem

$$
\begin{gathered}
\min _{\mathbf{b}, \mathbf{F}} \operatorname{tr}\left(\mathbf{F}\left(\mathbf{H b b}^{H} \mathbf{H}^{H}+\mathbf{I}_{N_{\mathbf{r}}}\right) \mathbf{F}^{H}\right)+\mathbf{b}^{H} \mathbf{b} \\
\text { s.t. }\left[1+\left(\mathbf{G}_{i} \mathbf{F H b}\right)^{H}\left(\mathbf{G}_{i} \mathbf{F} \mathbf{F}^{H} \mathbf{G}_{i}^{H}+\mathbf{I}_{N_{\mathrm{d}}}\right)^{-1} \mathbf{G}_{i} \mathbf{F H b}\right]^{-1} \\
\leq \varepsilon_{i}, \quad i=1, \cdots, L .
\end{gathered}
$$

It can be shown similar to [26] that the optimal transmit beamformer and relay precoding matrix as the solution to the problem (37) has the generic structure of $\mathbf{b}=\mathbf{v}_{\mathrm{h}, 1} \sqrt{P_{1}}$ and $\mathbf{F}=\mathbf{f u}_{\mathrm{h}, 1}^{H}$, respectively, where $P_{1}>0$ is the power required at the transmitter for multicasting a single data stream and remains to be optimized.

Based on the optimal structure of $\mathbf{b}$ and $\mathbf{F}$, and using (35), the QoS-constrained problem (37) can be equivalently converted to the following problem

$$
\begin{array}{ll}
\min _{P_{1}, \mathbf{f}} & P_{1}+\left(P_{1} \lambda_{\mathrm{h}, 1}^{2}+1\right) \mathbf{f}^{H} \mathbf{f} \\
\text { s.t. } & \frac{P_{1} \lambda_{\mathrm{h}, 1}^{2} \mathbf{f}^{H} \mathbf{G}_{i}^{H} \mathbf{G}_{i} \mathbf{f}}{\mathbf{f}^{H} \mathbf{G}_{i}^{H} \mathbf{G}_{i} \mathbf{f}+1} \geq \eta_{i}, \quad i=1, \cdots, L
\end{array}
$$

where $\eta_{i} \triangleq \frac{1}{\varepsilon_{i}}-1$ can be viewed as the minimal SNR required at the $i$ th receiver. The problem (38) is still nonconvex. However, it can be seen from (38b) that for a given $P_{1}$, there is $\mathbf{f}^{H} \mathbf{G}_{i}^{H} \mathbf{G}_{i} \mathbf{f} \geq \beta_{i}$ for $\beta_{i} \triangleq \frac{\eta_{i}}{P_{1} \lambda_{\mathrm{h}, 1}^{2}-\eta_{i}}>0, i=1, \cdots, L$. Thus, for a given $P_{1}$, the problem (38) reduces to

$$
\begin{aligned}
& \min _{\mathbf{f}} \mathbf{f}^{H} \mathbf{f} \\
& \text { s.t. } \mathbf{f}^{H} \mathbf{G}_{i}^{H} \mathbf{G}_{i} \mathbf{f} \geq \beta_{i}, \quad i=1, \cdots, L .
\end{aligned}
$$

The problem (39) is equivalent to the minimal transmission power beamforming problem for a single-hop multicasting system [3]. Note that the problem (39) can be solved using standard SDR techniques at a complexity order that is at most $\mathcal{O}\left(\left(N_{\mathrm{r}}^{2}+L\right)^{3.5}\right)[3]$.

Now we show some insights on the optimal $P_{1}$ by considering the objective function (38a). Obviously, it can be seen from the definition of $\beta_{i}$ that $P_{1} \lambda_{\mathrm{h}, 1}^{2}>\eta_{i}, i=1, \cdots, L$. In other words, the lower-bound of the feasible region of $P_{1}$ is $\max _{i}\left(\eta_{i}\right) / \lambda_{\mathrm{h}, 1}^{2}$. When $P_{1}$ is very close to its lower bound $\eta_{M} / \lambda_{\mathrm{h}, 1}^{2}, \beta_{M}=\frac{\eta_{M}}{P_{1} \lambda_{\mathrm{h}, 1}^{2}-\eta_{M}}>0$ is a very large number, where $M \triangleq \arg \max _{i}\left(\eta_{i}\right)$. As a result, $\mathbf{f}^{H} \mathbf{f}$ as the solution to the problem (39) is a large number, leading to a large value of (38a). When $P_{1}$ increases from $\eta_{M} / \lambda_{\mathrm{h}, 1}^{2}, \beta_{M}$ decreases, and the value of (38a) starts to decrease since the decrease of $\mathbf{f}^{H} \mathbf{f}$ dominates the increase of $P_{1}$. The value of (38a) keeps decreasing as $P_{1}$ increases till a 'turning point' where the increase of $P_{1}$ starts to dominate the decrease of $\mathbf{f}^{H} \mathbf{f}$. After such turning point, the value of (38a) increases monotonically as $P_{1}$ increases. To validate the analysis, a plot of the objective function (38a) over the range of feasible values of $P_{1}$ is generated in Fig. 2 for the case of $L=6$, $N_{\mathrm{s}}=6, N_{\mathrm{r}}=3, N_{\mathrm{d}}=3, \lambda_{\mathrm{h}, 1}=1.5345$, and $\eta_{i}=\eta$ is set to be $20 \mathrm{~dB}$. Here for each $P_{1}$, the problem (39) is solved to obtain the optimal f. It can be observed from Fig. 2 that the objective function (38a) is a unimodal function of $P_{1}$. An efficient method of locating the minimal value of a unimodal function is the golden section searching (GSS) algorithm [27]. Hence, the optimal $P_{1}$ for the problem (38) can be obtained by applying the GSS technique.

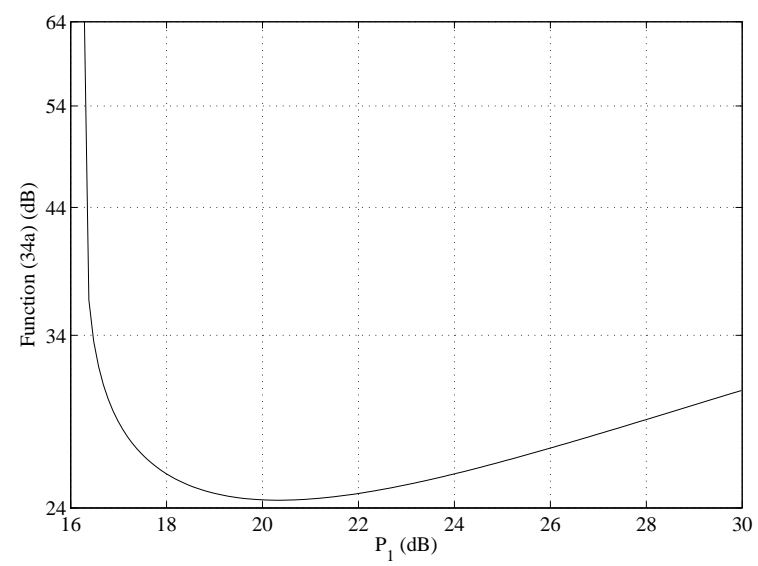

Fig. 2. Function (34a) versus $P_{1} . L=6, N_{\mathrm{s}}=6, N_{\mathrm{r}}=3, N_{\mathrm{d}}=3$, $\lambda_{\mathrm{h}, 1}=1.5345, \eta_{i}=\eta=20 \mathrm{~dB}$. 
Now the problem (38) can be solved through an iterative approach. For a fixed $P_{1}$, the problem (39) is solved to obtain $\mathrm{f}$, then $P_{1}$ is updated based on value of (38a) using the GSS technique. Then the problem (39) is solved again to update $\mathbf{f}$ according to the new $P_{1}$. This iterative procedure continues till the convergence of the GSS algorithm. Note that the solution of $P_{1}$ and $\mathbf{f}$ based on the GSS technique and solving the problem (39) with the SDR technique provides a suboptimal solution to the problem (38).

\section{NUMERICAL EXAMPLES}

In this section, we study the performance of the proposed two-hop multicasting MIMO relay optimization algorithms through numerical simulations. The transmitter, relay node, and receivers are equipped with $N_{\mathrm{s}}, N_{\mathrm{r}}$, and $N_{\mathrm{d}}$ antennas, respectively. We simulate a flat Rayleigh fading environment where the channel matrices have entries with zero mean and variances $1 / N_{\mathrm{s}}$ and $1 / N_{\mathrm{r}}$, for $\mathbf{H}$ and $\mathbf{G}_{i}, i=1, \cdots, L$, respectively. All simulation results are averaged over 500 independent channel realizations.

We compare the performance of the proposed min-max MSE algorithm in Section III-A with the naive amplify-andforward (NAF) algorithm and the pseudo match-and-forward (PMF) algorithm in terms of both MSE and BER. For the NAF scheme, we use

$$
\mathbf{B}=\sqrt{P_{\mathrm{s}} / N_{\mathrm{s}}} \mathbf{I}_{N_{\mathrm{s}}}, \quad \mathbf{F}=\sqrt{P_{\mathrm{r}} / \operatorname{tr}\left(\mathbf{H B B}^{H} \mathbf{H}^{H}+\mathbf{I}_{N_{\mathrm{r}}}\right)} \mathbf{I}_{N_{\mathrm{r}}} .
$$

For the PMF algorithm, the same $\mathbf{B}$ in the NAF algorithm is taken and

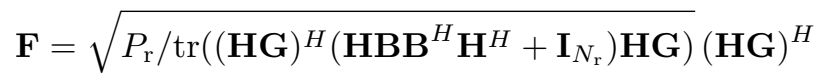

where we randomly pick $\mathbf{G}$ from among the relay-receiver channels $\mathbf{G}_{i}, i=1, \cdots, L$. Both the NAF and the PMF algorithms adopt the MMSE receiver at all receivers.

In the first example, we compare the performance of the proposed iterative and simplified algorithms with the other two approaches in terms of the MSE normalized by the number of data streams (NMSE) for $L=2$ and $N_{\mathrm{b}}=N_{\mathrm{s}}=N_{\mathrm{r}}=$ $N_{\mathrm{d}}=3$. Fig. 3 shows the MSE performance of all tested algorithms versus $P_{\mathrm{s}}$ with $P_{\mathrm{r}}=20 \mathrm{~dB}$. For the simplified algorithm in Section III.A.2, we plot the NMSE of the user with the worst channel (Worst) and the average of all the users (Avg.) along with the MSE upper-bound (UB), which is (19a). For the iterative algorithm in Section III.A.1, the NMSE of the worst channel is shown. Our results clearly demonstrate the better performance of the proposed joint transmitter and relay optimization algorithms. It can be seen that the proposed iterative algorithm consistently yield the lowest worstuser MSE over the entire $P_{\mathrm{S}}$ region. The worst-user MSE is always better than the MSE upper-bound. The NAF and PMF algorithms have much higher MSE compared with the proposed scheme even with very high transmission power. It can also be observed from Fig. 3 that the iterative and simplified algorithms have a similar worst-user MSE performance (curves marked with ' $*$ ' and ' $\times$ ' respectively), even at low SNRs. This indicates that the (moderately) high first-hop SNR assumption in the simplified algorithm introduces negligible performance loss in the whole SNR range in comparison to the iterative design. Note that the computational complexity of the simplified algorithm is less than the complexity of one iteration of the iterative algorithm, making it very attractive for practical MIMO relay multicasting systems. We will focus on the simplified algorithm in the following simulation examples.

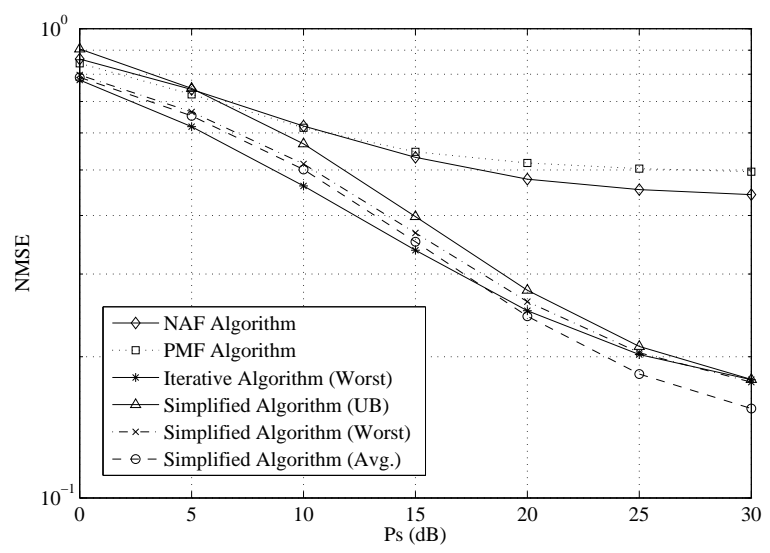

Fig. 3. Example 1: Normalized MSE versus $P_{\mathrm{s}} . L=2, N_{\mathrm{b}}=N_{\mathrm{s}}=N_{\mathrm{r}}=$ $N_{\mathrm{d}}=3, P_{\mathrm{r}}=20 \mathrm{~dB}$

In the second example, we compare the MSE performance of the simplified algorithm for different number of receivers. We set $N_{\mathrm{b}}=N_{\mathrm{s}}=N_{\mathrm{r}}=N_{\mathrm{d}}=3$. Fig. 4 shows the MSE upper-bound and the worst-user MSE of the simplified algorithm versus $P_{\mathrm{r}}$ with $P_{\mathrm{s}}=20 \mathrm{~dB}$. It can be clearly seen from Fig. 4 that as the number of receivers increases, the MSE upper-bound and the worst-user MSE keep increasing. This is reasonable since it is more likely to find a worse relay-receiver channel among the increased number of users and we choose the worst-user MSE as the objective function.

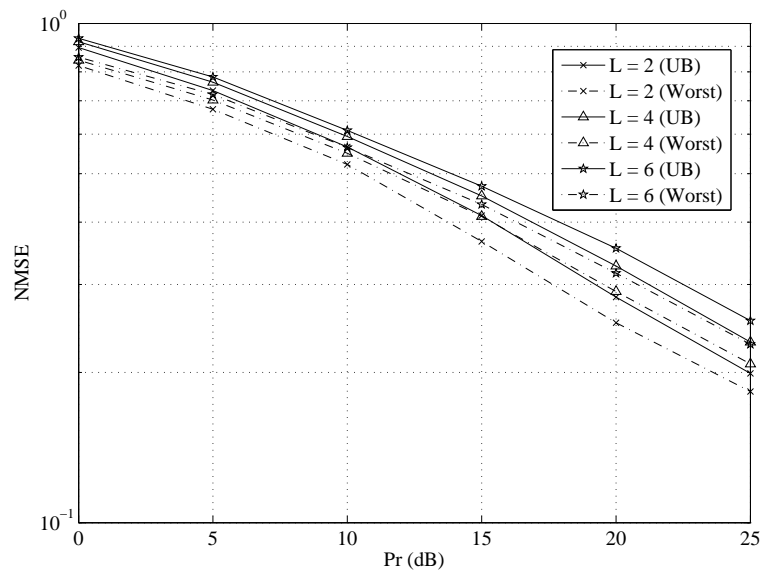

Fig. 4. Example 2: Normalized MSE versus $P_{\mathrm{r}}$. Varying number of receivers, $N_{\mathrm{b}}=N_{\mathrm{s}}=N_{\mathrm{r}}=N_{\mathrm{d}}=3, P_{\mathrm{s}}=20 \mathrm{~dB}$.

In the next example, we compare the performance of the simplified min-max MSE algorithm with the NAF and PMF algorithms in terms of BER. QPSK signal constellations are used to modulate the transmitted signals. We set $L=2$, $N_{\mathrm{b}}=2, N_{\mathrm{s}}=4, N_{\mathrm{r}}=2, N_{\mathrm{d}}=4$, and multicast 
$N_{\mathrm{b}} \times 1000$ randomly generated bits from the transmitter in each channel realization. Fig. 5 shows the BER performance of all tested algorithms versus $P_{\mathrm{s}}$ with $P_{\mathrm{r}}=20 \mathrm{~dB}$. It can be seen from Fig. 5 that the proposed simplified joint transmitter and relay optimization algorithm obtains the lowest BER compared with the other approaches. Even the worst-user BER of the proposed simplified algorithm is always much better than that of the NAF and the PMF schemes.

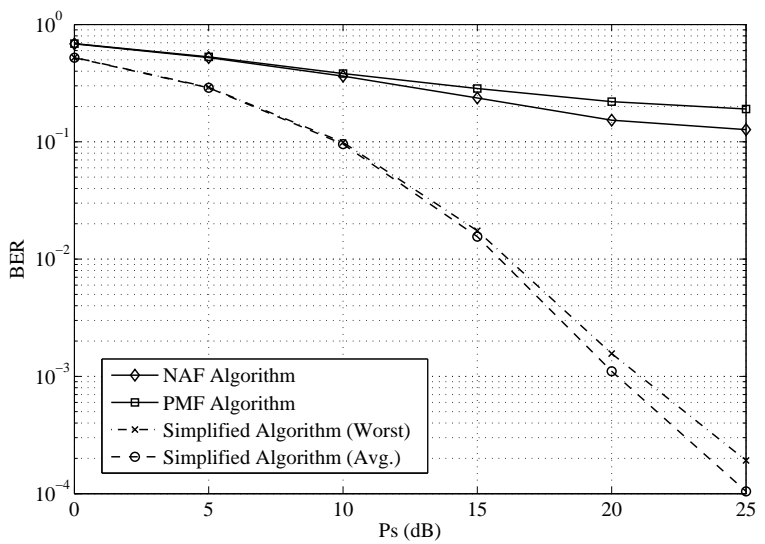

Fig. 5. Example 3: BER versus $P_{\mathrm{s}} . L=2, N_{\mathrm{b}}=2, N_{\mathrm{s}}=4, N_{\mathrm{r}}=2$, $N_{\mathrm{d}}=4, P_{\mathrm{r}}=20 \mathrm{~dB}$.

In the next example, we compare the average- and the worse-user BERs of the simplified algorithm for different number of receivers. This time we set $N_{\mathrm{b}}=2, N_{\mathrm{s}}=4$, $N_{\mathrm{r}}=2$, and $N_{\mathrm{d}}=4$. Fig. 6 shows the BER performance of the simplified algorithm versus $P_{\mathrm{s}}$ with $P_{\mathrm{r}}=20 \mathrm{~dB}$ for different number of receivers. It can be clearly seen from Fig. 6 that as we increase the number of receivers, the worstuser BER keeps increasing which is analogous to the MSE performance that we observed in Fig. 4. However, the average BERs of the users are almost similar for different number of receivers.

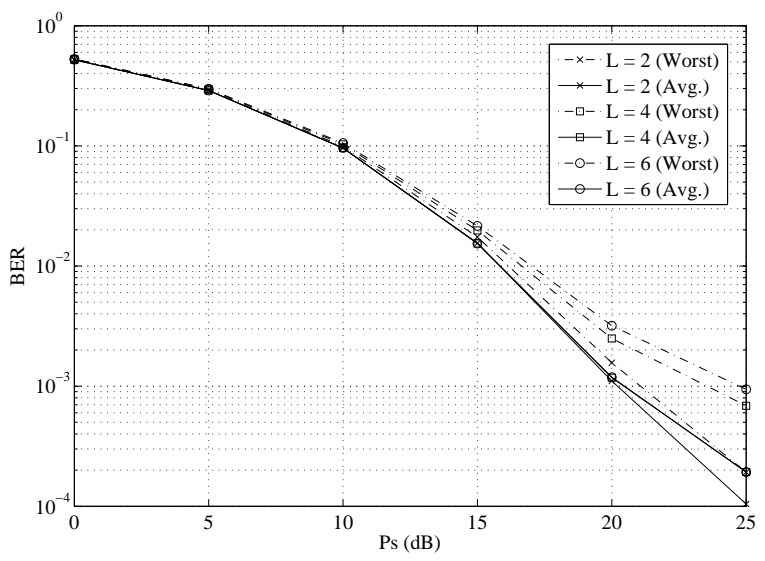

Fig. 6. Example 4: BER versus $P_{\mathrm{s}}$. Varying number of receivers, $N_{\mathrm{b}}=2$, $N_{\mathrm{s}}=4, N_{\mathrm{r}}=2, N_{\mathrm{d}}=4, P_{\mathrm{r}}=20 \mathrm{~dB}$.

In the fifth example, we study the performance of the total power minimization algorithm proposed in Section III-B for different number of receivers. For simplicity, we assume $\varepsilon_{i}=\varepsilon, i=1, \cdots, L$. Fig. 7 shows the total network transmission power versus the target MSE threshold $\varepsilon$ for $N_{\mathrm{b}}=N_{\mathrm{s}}=N_{\mathrm{r}}=N_{\mathrm{d}}=3$. Here the UB refers to (28a). From Fig. 7, it is obvious that more transmission power is required to multicast to a larger number of receivers assuring the same minimal MSE threshold at each receiver. The reason is that the proposed algorithm applies transmission power to satisfy the MSE requirement at all receivers. As the number of users increases, more transmission power is needed to combat the worst relay-receiver channel.

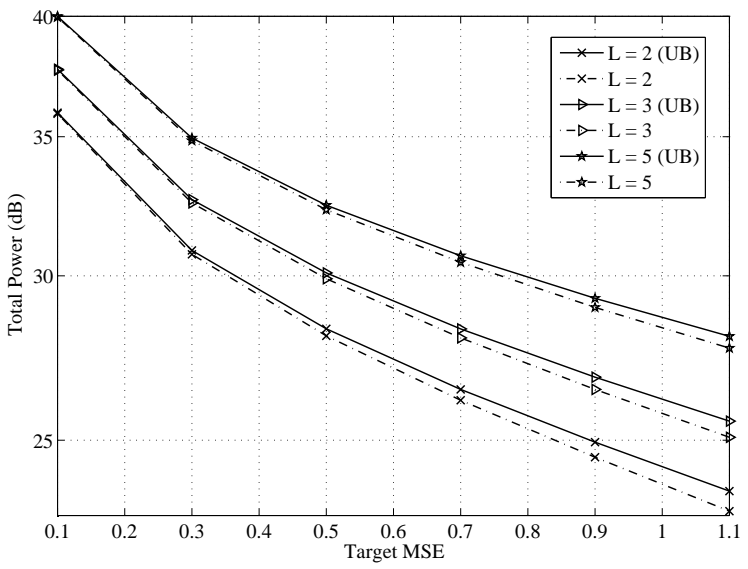

Fig. 7. Example 5: Total power versus target MSE $\varepsilon$. Varying number of receivers, $N_{\mathrm{b}}=N_{\mathrm{s}}=N_{\mathrm{r}}=N_{\mathrm{d}}=3$.

In the next two examples, we study the MI performance of the proposed single data stream two-hop multicasting MIMO relay algorithm developed in Section IV-A. The MI of the transmitter-relay- $i$ th receiver channel is calculated based on (35) as $\log _{2}\left(1+P_{\mathrm{s}} \lambda_{\mathrm{h}, 1}^{2}-\frac{P_{\mathrm{s}} \lambda_{\mathrm{h}, 1}^{2}}{\mathbf{f}^{H} \mathbf{G}_{i}^{H} \mathbf{G}_{i} \mathbf{f}+1}\right)$. Firstly, the average $\mathrm{MI}$ and the worst user MI of the proposed algorithm versus $P_{\mathrm{s}}$ are shown in Fig. 8 for $L=6, N_{\mathrm{s}}=6, N_{\mathrm{r}}=3, N_{\mathrm{d}}=3$, and $P_{\mathrm{r}}=20 \mathrm{~dB}$. From Fig. 8, we can see that even the worstchannel user can have a comparable MI performance with the average MI. In Fig. 9, the MI performance of the proposed algorithm versus the number of receivers $L$ is plotted with $N_{\mathrm{s}}=6, N_{\mathrm{r}}=3, N_{\mathrm{d}}=3$, and $P_{\mathrm{s}}=P_{\mathrm{r}}=20 \mathrm{~dB}$. Note that with the increasing number of receivers, the MI keeps decreasing as is also observed in [4]. It can also be seen from Fig. 9 that the worst user MI decreases slowly with increasing $L$. It has been shown in [4] that the multicast rate converges to zero when the number of users $L$ approaches to infinity.

In the last example, the total powers required for multicasting a single data stream among different number of receivers are compared. We use the GSS algorithm [27] to find the optimal $P_{1}$ for each target SNR threshold. The optimal relay precoding matrix is obtained by solving the problem (38) as is described in Section IV-B. For simplicity, we assume $\eta_{i}=\eta$, $i=1, \cdots, L$. Fig. 10 illustrates the total powers required for $L=2,4$, and 6 versus target SNR threshold $\eta$ with $N_{\mathrm{s}}=6$, $N_{\mathrm{r}}=3$, and $N_{\mathrm{d}}=3$. Since $N_{\mathrm{b}}=1$ is a special case of the system in Section III.B, the performance of the algorithm 


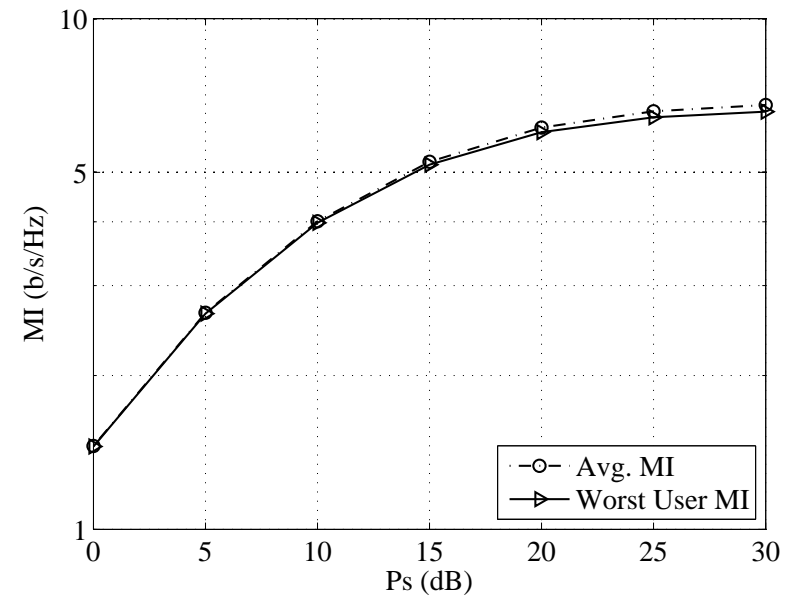

Fig. 8. Example 6: Per-user MI versus $P_{\mathrm{s}} . L=6, N_{\mathrm{b}}=1, N_{\mathrm{s}}=6$, $N_{\mathrm{r}}=3, N_{\mathrm{d}}=3, P_{\mathrm{r}}=20 \mathrm{~dB}$.

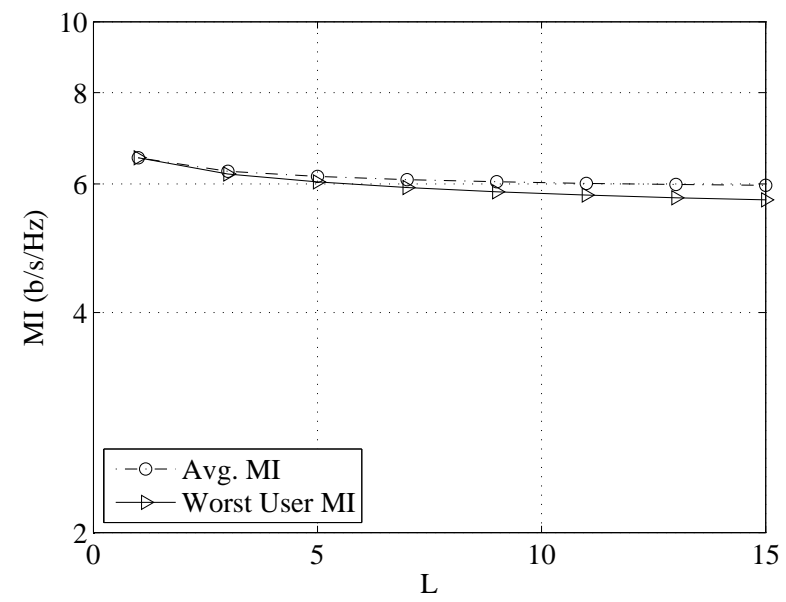

Fig. 9. Example 7: Per-user MI versus number of users $L . N_{\mathrm{b}}=1, N_{\mathrm{s}}=6$, $N_{\mathrm{r}}=3, N_{\mathrm{d}}=3, P_{\mathrm{s}}=P_{\mathrm{r}}=20 \mathrm{~dB}$.

based on solving the problem (28) is also shown in Fig. 10. It is notable that the gap among the total powers required for different number of receivers is small. This is because the power required by the multicasting system in this paper depends on the worst transmitter-relay-receiver channel. In this example, the number of antennas in each node is much larger than the number of data streams $\left(N_{\mathrm{b}}=1\right)$. This averages out the random channel leading to the so-called channel hardening effect. In other words, in the system in Fig. 10, the statistics of the worst channel among $L=4$ receivers is very close to that of the worst channel among $L=6$ receivers. Therefore, the power required by $L=4$ and $L=6$ systems is very close. It can also be seen from Fig. 10 that due to the high first-hop SNR approximation, the algorithm based on solving the problem (28) yields larger total power consumption compared with the algorithm based on solving the problem (38). However, since the problem (38) is solved through an iterative approach where the subproblem (39) is solved using the SDR technique in each iteration, the computational complexity involved in solving (38) is higher than that of the problem (28). Such performance-complexity tradeoff is useful in practical MIMO relay multicasting systems.

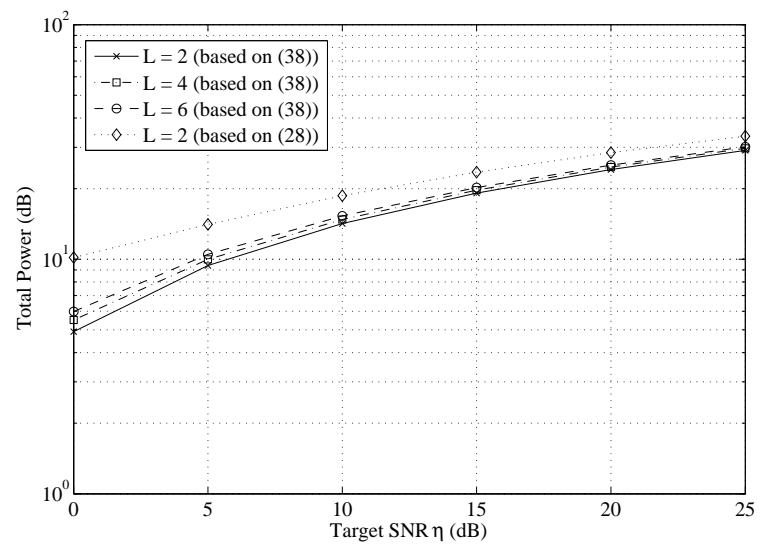

Fig. 10. Example 8: Total power versus target SNR $\eta$. Varying number of receivers, $N_{\mathrm{b}}=1, N_{\mathrm{s}}=6, N_{\mathrm{r}}=3, N_{\mathrm{d}}=3$.

\section{CONCLUSIONS}

We considered a two-hop multicasting MIMO relay system with multi-antenna nodes and proposed transmit and relay precoding matrices based on two design criteria. Firstly, the worst-case MSE was minimized subjecting to power constraints at the transmitter and the relay node. Secondly, we proposed a total network transmission power minimization strategy subjecting to QoS constraints. Under some mild approximation, we showed that the problems can be solved with a significantly smaller computational complexity. In addition, for the special case of single data stream multicasting, we established an interesting link between the relay precoding matrix in a two-hop MIMO multicasting system and the transmit beamformer in a single-hop multicasting system. Simulation results demonstrated that the proposed transmitter and relay design outperforms the existing techniques.

\section{ACKNOWLEDGMENT}

The authors would like to thank the editor and anonymous reviewers for their valuable comments and suggestions that improved the quality of the paper.

\section{REFERENCES}

[1] D. Tse and P. Viswanath, Fundamentals of Wireless Communication. Cambridge University Press, 2005.

[2] M. A. Khojastepour, A. Salehi-Golsefidi, and S. Rangarajan, "Towards an optimal beamforming algorithm for physical layer multicasting," in Proc. IEEE Inf. Theory Workshop, Oct. 16-20, 2011, pp. 395 -399.

[3] N. D. Sidiropoulos, T. N. Davidson, and Z.-Q. Luo, "Transmit beamforming for physical-layer multicasting," IEEE Trans. Signal Process., vol. 54, pp. 2239-2251, Jun. 2006.

[4] N. Jindal and Z.-Q. Luo, "Capacity limits of multiple antenna multicast," in Proc. IEEE ISIT, Seattle, USA, Jul. 09-14, 2006, pp. 1841-1845.

[5] S. Y. Park and D. J. Love, "Capacity limits of multiple antenna multicasting using antenna subset selection," IEEE Trans. Signal Process., vol. 56, pp. 2524-2534, Jun. 2008.

[6] S. Y. Park, D. J. Love, and D. H. Kim, "Capacity limits of multi-antenna multicasting under correlated fading channels," IEEE Trans. Commun., vol. 58, pp. 2002-2013, Jul. 2010. 
[7] E. Chiu and V. Lau, "Precoding design for multi-antenna multicast broadcast services with limited feedback," IEEE Systems Journal, vol. 4 pp. 550-560, Dec. 2010.

[8] Q. Li and W.-K. Ma, "Multicast secrecy rate maximization for MISO channels with multiple multi-antenna eavesdroppers," in Proc. IEEE ICC, Kyoto, Japan, Jun. 5-9, 2011.

[9] S. X. Wu and W.-K. Ma, "Multicast transmit beamforming using a randomize-in-time strategy," in Proc. IEEE ICASSP, Prague, Czech Republic, May 22-27, 2011, pp. 3376-3379.

[10] E. Matskani, N. D. Sidiropoulos, Z.-Q. Luo, and L. Tassiulas, "Efficient batch and adaptive approximation algorithms for joint multicast beamforming and admission control," IEEE Trans. Signal Process., vol. 57, pp. 4882-4894, Dec. 2009.

[11] N. Bornhorst and M. Pesavento, "An iterative convex approximation approach for transmit beamforming in multi-group multicasting," in Proc. IEEE 12th Int. Workshop Signal Process. Adv. Wireless Commun., San Francisco, USA, Jun. 26-29, 2011, pp. 426-430.

[12] D. Senaratne and C. Tellambura, "Beamforming for physical layer multicasting," in Proc. IEEE WCNC, Cancun, Mexico, Mar. 28-31, 2011, pp. 1776-1781.

[13] M. A. Khojastepour, A. Khajehnejad, K. Sundaresan, and S. Rangarajan, "Adaptive beamforming algorithms for wireless link layer multicasting," in Proc. IEEE PIMRC, Toronto, Canada, Sep. 11-14, 2011, pp. 1994 1998.

[14] M. Kaliszan, E. Pollakis, and S. Stańczak, "Efficient beamforming algorithms for MIMO multicast with application-layer coding," in Proc. IEEE ISIT, St. Petersburg, Russia, Jul. 31-Aug. 5, 2011, pp. 928-932.

[15] N. Bornhorst, M. Pesavento, and A. B. Gershman, "Distributed beamforming for multi-group multicasting relay networks," IEEE Trans. Signal Process., vol. 60, pp. 221-232, Jan. 2012.

[16] H. Xu, J. Jin, and B. Li, "YMMV: Multiple session multicast with MIMO," in Proc. IEEE GLOBECOM, Texas, USA, Dec. 5-9, 2011.

[17] H. Zhang, X. You, G. Wu, and H. Wang, "Cooperative multi-antenna multicasting for wireless networks," in IEEE GLOBECOM, Miami, FL, USA, Dec. 6-10, 2010.

[18] D. P. Palomar and Y. Jiang, MIMO Transceiver Design via Majorization Theory. now Publishers, 2007.

[19] S. M. Kay, Fundamentals of Statistical Signal Processing: Estimation Theory. Englewood Cilffs, NJ: Prentice Hall, 1993.

[20] Y. Rong, "Simplified algorithms for optimizing multiuser multi-hop MIMO relay systems," IEEE Trans. Commun., vol. 59, pp. 2896-2904, Oct. 2011

[21] C. Song, K.-J. Lee, and I. Lee, "MMSE based transceiver designs in closed-loop non-regenerative MIMO relaying systems," IEEE Trans. Wireless Commun., vol. 9, pp. 2310-2319, Jul. 2010.

[22] S. Boyd and L. Vandenberghe, Convex Optimization. Cambridge, U. K.: Cambridge University Press, 2004.

[23] M. Grant and S. Boyd, "Cvx: Matlab software for disciplined convex programming (web page and software)." http://cvxr.com/cvx, April, 2010.

[24] Y. Nesterov and A. Nemirovski, Interior Point Polynomial Algorithms in Convex Programming. Philadelphia, PA: SIAM, 1994.

[25] Y. Rong, X. Tang, and Y. Hua, "A unified framework for optimizing linear non-regenerative multicarrier MIMO relay communication systems," IEEE Trans. Signal Process., vol. 57, pp. 4837-4851, Dec. 2009.

[26] Y. Rong, "Multi-hop non-regenerative MIMO relays - QoS considerations," IEEE Trans. Signal Process., vol. 59, pp. 290-303, Jan. 2011.

[27] W. H. Press, S. A. Teukolsky, W. T. Vetterling, and B. P. Flannery, Numerical Recipes: The Art of Scientific Computing. Cambridge University Press, New York, 2007.

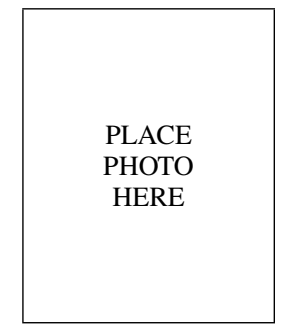

Yue Rong (S'03-M'06-SM'11) received the Ph.D. degree (summa cum laude) in electrical engineering from the Darmstadt University of Technology, Darmstadt, Germany, in 2005.

He was a Post-Doctoral Researcher with the Department of Electrical Engineering, University of California, Riverside, from February 2006 to November 2007. Since December 2007, he has been with the Department of Electrical and Computer Engineering, Curtin University, Bentley, Australia, where he is currently an Associate Professor. His current research interests include signal processing for communications, wireless communications, applications of linear algebra and optimization methods, and statistical and array signal processing.

Dr. Rong was a recipient of the Best Paper Award at the 2011 International Conference on Wireless Communications and Signal Processing, the Best Paper Award at the 2010 Asia-Pacific Conference on Communications, and the Young Researcher of the Year Award of the Faculty of Science and Engineering at Curtin University in 2010. He is an Editor of the IEEE WIRELESS COMMUNICATIONS LETTERS, a Guest Editor of the IEEE JOURNAL on Selected AREas in Communications special issue on theories and methods for advanced wireless relays, and was a TPC Member for the IEEE ICC, WCSP, IWCMC, and ChinaCom.

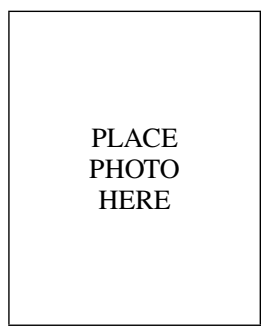

Muhammad R. A. Khandaker (S'10) received the Bachelor of Science (Honours) degree in Computer Science and Engineering from Jahangirnagar University, Dhaka, Bangladesh in 2006 and Master of Science in Telecommunications Engineering from East West University, Dhaka, Bangladesh in 2007. He is currently working toward the Ph.D. degree at the Department of Electrical and Computer Engineering, Curtin University, Australia.

Mr. Khandaker started his career as a Jr. Hardware Design Engineer in Visual Magic Corporation Limited (VMCL-BD) in November 2005. He joined as a Lecturer in the Department of Computer Science and Engineering, IBAIS University, Dhaka, Bangladesh in January 2006. In November 2007, he joined as a Lecturer in the Department of Information and Communication Technology of Mawlana Bhasani Science and Technology University, Bangladesh. Finally, he joined as a Lecturer in the Institute of Information Technology, Jahangirnagar University, Dhaka, Bangladesh in January 2008.

He was awarded Curtin International Postgraduate Research Scholarship (CIPRS) for his PhD study in 2009. He also received the Best Paper Award at the 16th Asia-Pacific Conference on Communications, Auckland, New Zealand, 2010. 